\title{
Connectedness of the theory of non-surjective injections
}

\author{
by
}

\author{
S. Świerczkowski (Muscat)
}

\begin{abstract}
It is shown that the first-order theory $\mathbf{T}$ of a non-surjective injection (of the universe into itself) is connected. By a criterion for connectedness, due to J. Mycielski, one reduces this result to the following theorem: If $\mathfrak{A}, \mathfrak{B}$ are any structures and $\varepsilon$ is a definable equivalence relation on their disjoint product $\mathfrak{A} \dot{\times} \mathfrak{B}$ such that $(\mathfrak{A} \dot{\times} \mathfrak{B}) / \varepsilon$ is a model of $\mathbf{T}$, then either $\mathfrak{A}$ has the property that for some finite structure $\mathfrak{S}$ and a definable equivalence relation $\simeq$ on $\mathfrak{S} \dot{\times} \mathfrak{A},(\mathfrak{S} \dot{\times} \mathfrak{A}) / \simeq$ is a model of $\mathbf{T}$, or the analogous property holds for $\mathfrak{B}$.
\end{abstract}

This paper has been triggered off by Mycielski's remark that every wellknown first-order theory of a simple nature should be connected. By now the list of theories for which this metamathematical conjecture has been verified has grown quite long (see [MPS], [SS]); the present paper extends it further. Since the connectedness of theories having finite models is easily derived from the basic definitions, we thought it worthwhile to take some very simple and natural theory that has no finite model and to show that it is connected. For this we chose the theory $\mathbf{T}$ of a non-surjective injection of the universe into itself. The proof that $\mathbf{T}$ is connected is presented below. It turned out surprisingly long and complicated. A large part is devoted to a detailed study of equivalence relations on disjoint products. This study trivializes if the given equivalence relation coincides with equality, to the effect of a considerable simplification of the whole proof. However, one may not impose a requirement that the relation of equality in $\mathbf{T}$ should be interpreted by equality in a structure, since many interesting results of the theory of interpretability owe their existence just to the possibility of interpreting equality by arbitrary equivalence relations (see [MPS]).

Problem. Is the "dual" theory of a non-injective surjection connected?

Acknowledgements. Special thanks are due to Alan Stern for pointing

1991 Mathematics Subject Classification: Primary 03F25. 
out faults in an earlier version of this proof and for his suggestions which led to a global improvement of the presentation.

\section{Statement of the main result}

1.1. We shall assume that the reader knows the definition of interpretability (local, multi-dimensional and with parameters) as proposed by Jan Mycielski (see [M], also [MPS], [S]). We recall that in this definition the theories are not supposed to have function symbols (so, if there are any, they must be replaced by relation symbols representing the graphs of the functions). Thus all structures will be assumed purely relational. If $\mathfrak{C}$ is such a structure and a first-order theory $T$ interprets 1-dimensionally in $\mathrm{Th}(\mathfrak{C})$, then we shall simply say that $T$ interprets in $\mathfrak{C}$, or that $\mathfrak{C}$ interprets $T$. We shall denote by $\mathbf{T}$ the theory of a non-surjective injection of the universe into itself. Then $\mathbf{T}$ interprets in $\mathfrak{C}$ iff there is a $\mathfrak{C}$-definable equivalence relation $\varepsilon$ on the universe $C$ of $\mathfrak{C}$ (interpreting the equality in $\mathbf{T}$ ) and a $\mathfrak{C}$-definable binary relation $r$ on $C$ which induces the graph of a non-surjective injection of $C / \varepsilon$ (= the set of $\varepsilon$-equivalence classes) into itself. In other words, the following sentences are valid in $\mathfrak{C}$ :

$$
\begin{aligned}
& \forall x \exists y(r(x, y)), \\
& \exists z \forall x(\neg r(x, z)), \\
& \forall x \forall y \forall x^{\prime} \forall y^{\prime}\left(r(x, y) \wedge r\left(x^{\prime}, y^{\prime}\right) \rightarrow\left(\varepsilon\left(x, x^{\prime}\right) \leftrightarrow \varepsilon\left(y, y^{\prime}\right)\right)\right), \\
& \forall x \forall y \forall x^{\prime} \forall y^{\prime}\left(\varepsilon\left(x, x^{\prime}\right) \wedge \varepsilon\left(y, y^{\prime}\right) \rightarrow\left(r(x, y) \leftrightarrow r\left(x^{\prime}, y^{\prime}\right)\right)\right) .
\end{aligned}
$$

If these sentences are valid in $\mathfrak{C}$, we say that the pair $(\varepsilon, r)$ interprets $\mathbf{T}$.

Recall that a theory $T$ is called connected if $T$ interprets in a disjoint union of two theories $T_{1} \dot{\cup} T_{2}$ only when $T$ interprets in $T_{1}$ or in $T_{2}$. We shall deduce the connectedness of $\mathbf{T}$ from the following criterion due to Jan Mycielski ([MPS], p. 52).

1.2. TheOREM. A finitely axiomatizable theory $T$ is connected iff for all structures $\mathfrak{A}$ and $\mathfrak{B}$,

( $T$ interprets in the disjoint product $\mathfrak{A} \dot{\times} \mathfrak{B})$

$$
\Rightarrow(T \text { interprets in } \operatorname{Th}(\mathfrak{A}) \text { or in } \operatorname{Th}(\mathfrak{B})) \text {. }
$$

In order to apply this criterion, we prove here the following theorem for the theory $\mathbf{T}$ of a non-surjective injection.

1.3. TheOrem. If $\mathbf{T}$ interprets in a disjoint product $\mathfrak{C}=\mathfrak{A} \dot{\times} \mathfrak{B}$ then there is a finite structure $\mathfrak{S}$ such that $\mathbf{T}$ interprets in $\mathfrak{S} \dot{\times} \mathfrak{A}$ or in $\mathfrak{S} \dot{\times} \mathfrak{B}$.

This will suffice to deduce that $\mathbf{T}$ is connected. To see this, recall that the chapter $|T|$ of a first order theory $T$ is the class of those theories which 
both interpret and are interpretable in $T$. The set of all chapters forms a lattice with respect to the partial order $\leq$ given by

$$
|T| \leq|Q| \quad \text { iff } \quad T \text { interprets in } Q .
$$

Also, $|\operatorname{Th}(\mathfrak{A} \dot{\times} \mathfrak{B})|$ is equal to the lattice join $|\operatorname{Th}(\mathfrak{A})| \vee|\operatorname{Th}(\mathfrak{B})|$ (see [MTS], $[\mathrm{S}])$. So now suppose that Theorem 1.3 is established, i.e., we have deduced from the interpretability of $\mathbf{T}$ in $\mathfrak{A} \dot{\times} \mathfrak{B}$ that $\mathbf{T}$ interprets in $\mathfrak{S} \dot{\times} \mathfrak{A}$ or in $\mathfrak{S} \dot{\times} \mathfrak{B}$, for some finite $\mathfrak{S}$. If $\mathbf{T}$ interprets in $\mathfrak{S} \dot{\times} \mathfrak{A}$ then this structure must be infinite, and since $\mathfrak{S}$ is finite, we deduce that $\mathfrak{A}$ is infinite. Therefore $|\operatorname{Th}(\mathfrak{S})| \leq|\operatorname{Th}(\mathfrak{A})|$ and it follows that

$$
|\mathbf{T}| \leq|\operatorname{Th}(\mathfrak{S} \dot{\times} \mathfrak{A})|=|\operatorname{Th}(\mathfrak{S})| \vee|\operatorname{Th}(\mathfrak{A})| \leq|\operatorname{Th}(\mathfrak{A})| \vee|\operatorname{Th}(\mathfrak{A})|=|\operatorname{Th}(\mathfrak{A})|,
$$

i.e., $\mathbf{T}$ interprets in $\operatorname{Th}(\mathfrak{A})$. In the same way we deduce from $|\mathbf{T}| \leq$ $|\operatorname{Th}(\mathfrak{S} \dot{\times} \mathfrak{B})|$ that $|\mathbf{T}| \leq|\operatorname{Th}(\mathfrak{B})|$. So we see that Mycielski's criterion 1.2 is satisfied, whence $\mathbf{T}$ is connected.

The next section is general; it concerns an arbitrary definable equivalence relation $\varepsilon$ on a disjoint product. The main result (Theorem 2.3) describes $\varepsilon$ by a finite system of finite groups acting coordinatewise and without fixed points on disjoint rectangles.

\section{Equivalence relations in disjoint products}

2.1. It is clear that whenever a theory $T$ is interpreted in a disjoint product $\mathfrak{A} \dot{\times} \mathfrak{B}$ (say, if one wishes to show that $T$ is connected, using Theorem 1.2), there will be a need to consider the definable equivalence relation $\varepsilon$ on $A \times B$ which interprets the equality in $T$. Thus the general description of such an equivalence, obtained in this section, could possibly be of use in establishing the connectedness of theories other than $\mathbf{T}$ (we return to the specific theory $\mathbf{T}$ in Section 3).

Let $\mathfrak{A}$ and $\mathfrak{B}$ be given structures, and let $\mathfrak{C}$ denote the disjoint product $\mathfrak{A} \dot{\times} \mathfrak{B}$, i.e., $C=A \times B$ is the universe of $\mathfrak{C}$, while the defining relations are the cylinders over the $\mathfrak{A}$-definable relations in $\mathfrak{A}$ and over the $\mathfrak{B}$-definable relations in $\mathfrak{B}$. Let $\varepsilon$ be a $\mathfrak{C}$-definable equivalence relation $\varepsilon$ on $C$. We shall show that $\varepsilon$ determines naturally another equivalence relation $\bar{\varepsilon} \subseteq \varepsilon$ and we shall consider the relation $\widetilde{\varepsilon}$ induced by $\varepsilon$ on the quotient set $C / \bar{\varepsilon}$. It will be seen in the later sections that for proving Theorem 1.3, one can replace the interpreting pair $(\varepsilon, r)$ by $(\widetilde{\varepsilon}, \widetilde{r})$, where $\widetilde{r}=r / \bar{\varepsilon}$. The main properties of $\widetilde{\varepsilon}$ are listed in Theorem 2.3. To state it, we need first some definitions.

2.2. We shall say that a relation $s \subseteq C^{n}$ splits if there are relations $s_{A} \subseteq A^{n}$ and $s_{B} \subseteq B^{n}$ such that

$$
s\left(x_{1}, \ldots, x_{n}\right) \leftrightarrow s_{A}\left(u_{1}, \ldots, u_{n}\right) \wedge s_{B}\left(v_{1}, \ldots, v_{n}\right)
$$


for all $x_{i}=\left(u_{i}, v_{i}\right)$ in $C(i=1, \ldots, n)$. In this case we shall call $s_{A}$ and $s_{B}$ the projections of $s$, and say that $s$ is their product, writing $s=s_{A} \times s_{B}$. We note that if $\emptyset \neq s=s_{A} \times s_{B}$ then $s_{A}$ and $s_{B}$ are uniquely determined by $s$. If $s_{A}$ and $s_{B}$ are $\mathfrak{A}$-definable and $\mathfrak{B}$-definable, respectively, then we shall say that $s$ splits $\mathfrak{C}$-definably. It is well known that $s$ is $\mathfrak{C}$-definable iff $s \leftrightarrow \bigvee_{p=1}^{t} s^{(p)}$, where each $s^{(p)}$ is a $\mathfrak{C}$-definably splitting relation (see $[\mathrm{S}]$, Lemma 1.14). It follows that if $\emptyset \neq s=s_{A} \times s_{B}$ is $\mathfrak{C}$-definable then $s_{A}$ is $\mathfrak{A}$-definable and $s_{B}$ is $\mathfrak{B}$-definable. We shall abbreviate henceforth "CC-definable" to "definable".

We need some further notation. Let $P$ be a set and let $\eta$ be an equivalence relation on $P$. For each $x \in P$, the $\eta$-equivalence class of $x$ will be denoted by $x / \eta$, and if $D \subseteq P$ is a union of $\eta$-equivalence classes then we shall write $D / \eta=\{x / \eta: x \in D\}$. A relation $s \subseteq P^{n}$ will be called $\eta$-invariant if the validity of $s\left(x_{1}, \ldots, x_{n}\right)$ depends only on $x_{1} / \eta, \ldots, x_{n} / \eta$. It is clear that in such case $s$ induces in a natural way an $n$-ary relation on $P / \eta$, which we shall denote by $s / \eta$. Evidently each $t \subseteq(P / \eta)^{n}$ is induced in this way by a uniquely determined $\eta$-invariant $s \subseteq P^{n}$; instead of $t=s / \eta$, we shall also write $t \cdot \eta=s$.

Often we shall call the Cartesian product $P$ of two sets a rectangle, and in that case the two sets will be denoted by $P_{A}$ and $P_{B}$, and called the projections of $P$. If a rectangle $P$ is a subset of a product $X \times Y$ then $P_{A} \subseteq X$ and $P_{B} \subseteq Y$. Suppose $g$ is a map whose domain and range are rectangles: $P=$ $\operatorname{dom}(g), P^{\prime}=\operatorname{rng}(g)$. Then $g$ will be said to split if there are maps $g_{A}: P_{A} \rightarrow$ $P_{A}^{\prime}$ and $g_{B}: P_{B} \rightarrow P_{B}^{\prime}$ such that $g(u, v)=\left(g_{A}(u), g_{B}(v)\right)$ for all $(u, v) \in P$. We shall call $g_{A}$ and $g_{B}$ the projections of $g$, and write $g=g_{A} \times g_{B}$.

Suppose that $P \subseteq C=A \times B$ is a rectangle and $s$ is an equivalence relation on $P$ which splits (as a relation $s \subseteq C^{2}$ ). Then it is easy to see that the projections $s_{A}$ and $s_{B}$ are equivalence relations on $P_{A}$ and $P_{B}$, respectively; moreover, the $s$-equivalence classes are the products of $s_{A}$-equivalence classes by $s_{B}$-equivalence classes. Thus $P / s$ is also a rectangle, via the obvious identification

$$
P / s=P_{A} / s_{A} \times P_{B} / s_{B} .
$$

Let us show that $\varepsilon$ determines a finite partition $\overline{\mathcal{D}}_{\varepsilon}$ of $C$ into definable rectangles. Given $b \in B$, we can take the restriction of $\varepsilon$ to $A \times\{b\}$ and then project the obtained relation on $A$. This produces an equivalence relation $\varepsilon^{b}$ on $A$ given by

$$
\varepsilon^{b}\left(u, u^{\prime}\right) \leftrightarrow \varepsilon\left((u, b),\left(u^{\prime}, b\right)\right) \quad \text { for all } u, u^{\prime} \in A .
$$

Similarly, for each $a \in A$, we define the equivalence relation $\varepsilon^{a}$ on $B$ by

$$
\varepsilon^{a}\left(v, v^{\prime}\right) \leftrightarrow \varepsilon\left((a, v),\left(a, v^{\prime}\right)\right) \quad \text { for all } v, v^{\prime} \in B .
$$

Since $\varepsilon$ is definable, we have $\varepsilon \leftrightarrow \bigvee_{p=1}^{t} \varepsilon^{(p)}$, where each $\varepsilon^{(p)}$ is a definably 
splitting binary relation. Thus, for every $u, u^{\prime} \in A$,

$$
\varepsilon^{b}\left(u, u^{\prime}\right) \leftrightarrow \bigvee_{p=1}^{t}\left(\varepsilon_{A}^{(p)}\left(u, u^{\prime}\right) \wedge \varepsilon_{B}^{(p)}(b, b)\right),
$$

which shows that $\varepsilon^{b}$ is $\mathfrak{A}$-definable, and also that we get only finitely many distinct relations $\varepsilon^{b}$ as $b$ ranges over $B$. We shall denote by $B=B_{1} \dot{\cup} \ldots \dot{\cup} B_{n}$ the partition of $B$ such that $\varepsilon^{b^{\prime}}=\varepsilon^{b}$ iff $b^{\prime}$ and $b$ belong to the same $B_{j}$ $(j=1, \ldots, n)$. Obviously, each $B_{j}$ is $\mathfrak{B}$-definable.

Similarly, we get a partition of $A$ into $\mathfrak{A}$-definable subsets $A_{1}, \ldots, A_{m}$ such that $\varepsilon^{a^{\prime}}=\varepsilon^{a}$ iff $a^{\prime}$ and $a$ belong to the same set $A_{i}$. Let $\overline{\mathcal{D}}_{\varepsilon}$ be the partition of $C$ into the rectangles $A_{i} \times B_{j}(i=1, \ldots, m, j=1, \ldots, n)$. Thus each $\bar{D} \in \overline{\mathcal{D}}_{\varepsilon}$ is a rectangle $\bar{D}_{A} \times \bar{D}_{B}$, where $\bar{D}_{A}=A_{i}$ and $\bar{D}_{B}=B_{j}$, for some $i, j$.

Definition. Given any rectangle $\bar{D} \in \overline{\mathcal{D}}_{\varepsilon}$, we shall denote by $\bar{\varepsilon}^{D}$ the equivalence relation on $\bar{D}$ defined as follows: Choose any $(a, b) \in \bar{D}$, denote by $\bar{\varepsilon}_{A}^{D}$ the restriction of $\varepsilon^{b}$ to $\bar{D}_{A}$, by $\bar{\varepsilon}_{B}^{D}$ the restriction of $\varepsilon^{a}$ to $\bar{D}_{B}$, and put $\bar{\varepsilon}^{D}=\bar{\varepsilon}_{A}^{D} \times \bar{\varepsilon}_{B}^{D}$.

Clearly $\bar{\varepsilon}^{D}$ does not depend on the particular choice of $(a, b)$ in $\bar{D}$, and $\bar{\varepsilon}^{D} \subseteq \varepsilon$. Moreover, $\bar{\varepsilon}^{D}$ is a definably splitting equivalence relation on $\bar{D}$, whence the quotient set $D=\bar{D} / \bar{\varepsilon}^{D}$ is also a rectangle, namely

$$
D=D_{A} \times D_{B}, \quad \text { where } D_{A}=\bar{D}_{A} / \bar{\varepsilon}_{A}^{D} \text { and } D_{B}=\bar{D}_{B} / \bar{\varepsilon}_{B}^{D} \text {. }
$$

Notation. The following notation will be adhered to until the end of the paper:

$$
\begin{aligned}
& \bar{\varepsilon}=\bigvee\left\{\bar{\varepsilon}^{D}: \bar{D} \in \overline{\mathcal{D}}_{\varepsilon}\right\}=\text { disjoint union of the equivalence relations } \bar{\varepsilon}^{D} \text { on } \\
& \text { the rectangles } \bar{D}=A_{i} \times B_{j}, \\
& \mathbb{C}=C / \bar{\varepsilon}=\text { quotient set modulo the equivalence } \bar{\varepsilon}, \\
& \widetilde{\varepsilon}=\varepsilon / \bar{\varepsilon}=\text { equivalence relation induced by } \varepsilon \text { on the quotient set } \mathbb{C}, \\
& \mathcal{D}_{\varepsilon}=\left\{D: \bar{D} \in \overline{\mathcal{D}}_{\varepsilon}\right\}=\text { finite partition of } \mathbb{C} \text { into rectangles induced by the } \\
& \text { partition } \overline{\mathcal{D}}_{\varepsilon} \text { of } C .
\end{aligned}
$$

If $P$ is a rectangle which is contained in some $D \in \mathcal{D}_{\varepsilon}$ then we shall say that $P$ is a rectangle in $\mathbb{C}$.

A relation $t \subseteq \mathbb{C}^{n}$ will be called definable if $t \cdot \bar{\varepsilon} \subseteq C^{n}$ is definable. In particular, if $n=1$, we thus obtain the definition of a definable subset of $\mathbb{C}$. If $n=2$ and $t$ is the graph of a mapping $g$ (with $\operatorname{dom}(g), \operatorname{rng}(g) \subseteq \mathbb{C}$ ) then $g$ will be called definable iff $t$ is definable.

We can now state the main theorem of this section.

2.3. TheOREM. There exists a finite set $\mathcal{R}$ of definable disjoint rectangles in $\mathbb{C}$, and for every $R \in \mathcal{R}$, a finite group $G_{R}$ acting on $R$ (as a group of bijections $R \rightarrow R$ ) such that 
(1) Every $\widetilde{\varepsilon}$-equivalence class intersects exactly one $R \in \mathcal{R}$, and their intersection is a $G_{R}$-orbit.

(2) Every bijection $g: R \rightarrow R\left(R \in \mathcal{R}, g \in G_{R}\right)$ is definable, it splits, its projections $g_{A}: R_{A} \rightarrow R_{A}$ and $g_{B}: R_{B} \rightarrow R_{B}$ are bijective and they have no fixed points, unless $g$ is the unit of $G_{R}$.

The proof is given in Sections 2.4-2.14.

2.4. Lemma. Let $s \subseteq C^{2}$ be definable. Then $s \leftrightarrow \bigvee_{q=1}^{r} \bar{s}^{(q)}$, where each $\bar{s}^{(q)}$ splits definably and for every $q=1, \ldots, r, \operatorname{dom}\left(\bar{s}^{(q)}\right)$ and $\operatorname{rng}\left(\bar{s}^{(q)}\right)$ are rectangles contained in some $\bar{D}, \bar{D}^{\prime} \in \overline{\mathcal{D}}_{\varepsilon}$.

Proof. For each $\bar{D}, \bar{D}^{\prime} \in \overline{\mathcal{D}}_{\varepsilon}$ consider the relation $\eta^{D, D^{\prime}} \subseteq C^{2}$ given by $\eta^{D, D^{\prime}}(x, y) \leftrightarrow x \in \bar{D} \wedge y \in \bar{D}^{\prime}$. Then $\eta^{D, D^{\prime}}$ splits definably because, for $\bar{D}=A_{i} \times B_{j}$ and $\bar{D}^{\prime}=A_{k} \times B_{t}$,

$$
\eta^{D, D^{\prime}}\left(\left(x_{A}, x_{B}\right),\left(y_{A}, y_{B}\right)\right) \leftrightarrow\left(x_{A} \in A_{i} \wedge y_{A} \in A_{k}\right) \wedge\left(x_{B} \in B_{j} \wedge y_{B} \in B_{t}\right) .
$$

Clearly the disjunction of all $\eta^{D, D^{\prime}}$ is valid at each $(x, y) \in C^{2}$. By 2.2, $s \leftrightarrow \bigvee_{p=1}^{t} s^{(p)}$, where each $s^{(p)}$ splits definably. Thus

$$
s \leftrightarrow \bigvee\left\{s^{(p)} \wedge \eta^{D, D^{\prime}}: p=1, \ldots, t ; \bar{D}, \bar{D}^{\prime} \in \overline{\mathcal{D}}_{\varepsilon}\right\}
$$

Clearly $s^{(p)} \wedge \eta^{D, D^{\prime}}$ splits definably. Moreover, the domain of this relation is a rectangle contained in $\bar{D}$, and its range is a rectangle contained in $\bar{D}^{\prime}$.

2.5. To state the next lemma, we need more notation. Suppose $S, S^{\prime}$ are two sets and $\eta, \eta^{\prime}$ are equivalence relations on these sets. Then a relation $s \subseteq S \times S^{\prime}$ will be called $\left(\eta, \eta^{\prime}\right)$-invariant if the validity of $s\left(u, u^{\prime}\right)$ depends only on the equivalence classes $u / \eta$ and $u^{\prime} / \eta^{\prime}$. In that case $s$ induces in an obvious way a relation $t \subseteq S / \eta \times S^{\prime} / \eta^{\prime}$. Generalizing the notation of 2.2 , we shall write $s=\eta \cdot t \cdot \eta^{\prime}$.

Given two sets $\Delta, \Delta^{\prime}$ and an injective map $\gamma$, we shall call $\gamma$ a local injection from $\Delta$ to $\Delta^{\prime}$ (or on $\Delta$, if $\Delta=\Delta^{\prime}$ ) if $\operatorname{dom}(\gamma) \subseteq \Delta$ and $\operatorname{rng}(\gamma) \subseteq \Delta^{\prime}$.

We note that if $\gamma$ is a local injection on $\mathbb{C}$ whose domain and range are rectangles in $\mathbb{C}$ then it is meaningful to say that $\gamma$ splits (see 2.2).

2.6. Lemma. Suppose $\emptyset \neq s \subseteq C^{2}$ is a definable relation which is $\varepsilon$ invariant and satisfies

$$
s(x, y) \wedge s\left(x^{\prime}, y^{\prime}\right) \rightarrow\left(\varepsilon\left(x, x^{\prime}\right) \leftrightarrow \varepsilon\left(y, y^{\prime}\right)\right),
$$

for all $x, x^{\prime}, y, y^{\prime} \in C$. Then there is a finite set $\Gamma_{s}$ of local injections on $\mathbb{C}$ such that

(1) For every $x, y \in \mathbb{C}$,

$$
(s / \bar{\varepsilon})(x, y) \leftrightarrow \exists\left(\gamma \in \Gamma_{s}\right)(\gamma(x)=y) .
$$


(2) Each $\gamma \in \Gamma_{s}$ is definable, and its domain and range are rectangles in $\mathbb{C} ;$ moreover, $\gamma$ splits, and its projections $\gamma_{A}$ and $\gamma_{B}$ are both injective.

Remark. The assumptions of this lemma are satisfied if $s=\varepsilon$, or if $s$ is any relation such that $(\varepsilon, s)$ interprets the theory $\mathbf{T}$ (or, more generally, the theory of an injection).

Proof of the lemma. Let $s \leftrightarrow \bigvee_{q=1}^{r} s^{(q)}$, where each $s^{(q)}$ splits definably and for every $q=1, \ldots, r$ there are $\bar{D}, \bar{D}^{\prime} \in \overline{\mathcal{D}}_{\varepsilon}$ for which $\operatorname{dom}\left(s^{(q)}\right) \subseteq$ $\bar{D}$ and $\operatorname{rng}\left(s^{(q)}\right) \subseteq \bar{D}^{\prime}$. Such $s^{(q)}$ exist, by Lemma 2.4. Since $s \neq \emptyset$, we may assume that each $s^{(q)}$ is non-empty. We claim that, without loss of generality, we may also assume that for every $q, s_{A}^{(q)}$ is $\left(\bar{\varepsilon}_{A}^{D}, \bar{\varepsilon}_{A}^{D^{\prime}}\right)$-invariant and $s_{B}^{(q)}$ is $\left(\bar{\varepsilon}_{B}^{D}, \bar{\varepsilon}_{B}^{D^{\prime}}\right)$-invariant. Indeed, should this not be the case, we can replace $s_{A}^{(q)}$ and $s_{B}^{(q)}$ by $\bar{s}_{A}^{(q)}$ and $\bar{s}_{B}^{(q)}$, where

$$
\bar{s}_{A}^{(q)}(u, v) \leftrightarrow \exists u^{\prime} v^{\prime}\left(\bar{\varepsilon}_{A}^{D}\left(u, u^{\prime}\right) \wedge s_{A}^{(q)}\left(u^{\prime}, v^{\prime}\right) \wedge \bar{\varepsilon}_{A}^{D^{\prime}}\left(v^{\prime}, v\right)\right)
$$

for all $u, v \in A$, and $\bar{s}_{B}^{(q)}$ is defined similarly. Further, we can replace each $s^{(q)}$ by $\bar{s}^{(q)}=\bar{s}_{A}^{(q)} \times \bar{s}_{B}^{(q)}$. Let us check that $s \leftrightarrow \bigvee_{q=1}^{r} \bar{s}^{(q)}$. Since $s_{A}^{(q)} \rightarrow \bar{s}_{A}^{(q)}$ and $s_{B}^{(q)} \rightarrow \bar{s}_{B}^{(q)}$, we get $s^{(q)} \rightarrow \bar{s}^{(q)}$, so it will suffice to show that $\bar{s}^{(q)} \rightarrow s$. By the above definition of $\bar{s}^{(q)}$ and $\bar{\varepsilon}^{D}, \bar{\varepsilon}^{D^{\prime}} \subseteq \varepsilon$,

$$
\begin{aligned}
\bar{s}^{(q)}(x, y) & \rightarrow \exists x^{\prime} y^{\prime}\left(\bar{\varepsilon}^{D}\left(x, x^{\prime}\right) \wedge s^{(q)}\left(x^{\prime}, y^{\prime}\right) \wedge \bar{\varepsilon}^{D^{\prime}}\left(y^{\prime}, y\right)\right) \\
& \rightarrow \exists x^{\prime} y^{\prime}\left(\varepsilon\left(x, x^{\prime}\right) \wedge s\left(x^{\prime}, y^{\prime}\right) \wedge \varepsilon\left(y^{\prime}, y\right)\right),
\end{aligned}
$$

and the last formula implies $s(x, y)$, because $s$ is $\varepsilon$-invariant.

Thus, assuming henceforth the $\left(\bar{\varepsilon}_{A}^{D}, \bar{\varepsilon}_{A}^{D^{\prime}}\right)$-invariance of $s_{A}^{(q)}$ and the $\left(\bar{\varepsilon}_{B}^{D}, \bar{\varepsilon}_{B}^{D^{\prime}}\right)$-invariance of $s_{B}^{(q)}$, we see that $s^{(q)}$ is $\bar{\varepsilon}$-invariant, and that $s_{A}^{(q)}$ and $s_{B}^{(q)}$ induce relations

$$
\overline{\bar{s}}_{A}^{(q)} \subseteq D_{A} \times D_{A}^{\prime}, \quad \overline{\bar{s}}_{B}^{(q)} \subseteq D_{B} \times D_{B}^{\prime}
$$

(satisfying $s_{A}^{(q)}=\bar{\varepsilon}_{A}^{D} \cdot \overline{\bar{s}}_{A}^{(q)} \cdot \bar{\varepsilon}_{A}^{D^{\prime}}$ and $s_{B}^{(q)}=\bar{\varepsilon}_{B}^{D} \cdot \overline{\bar{s}}_{B}^{(q)} \cdot \bar{\varepsilon}_{B}^{D^{\prime}}$ ). Let us prove that $\overline{\bar{s}}_{A}^{(q)}, \overline{\bar{s}}_{B}^{(q)}$ are graphs of injections. For $\overline{\bar{s}}_{A}^{(q)}$ this means that for all $u, u^{\prime} \in \bar{D}_{A}$ and $v, v^{\prime} \in \bar{D}_{A}^{\prime}$,

$$
s_{A}^{(q)}(u, v) \wedge s_{A}^{(q)}\left(u^{\prime}, v^{\prime}\right) \rightarrow\left(\bar{\varepsilon}_{A}^{D}\left(u, u^{\prime}\right) \leftrightarrow \bar{\varepsilon}_{A}^{D^{\prime}}\left(v, v^{\prime}\right)\right) .
$$

To show that this is so, assume that $s_{A}^{(q)}(u, v)$ and $s_{A}^{(q)}\left(u^{\prime}, v^{\prime}\right)$ both hold. We have to deduce that then $\bar{\varepsilon}_{A}^{D}\left(u, u^{\prime}\right) \leftrightarrow \bar{\varepsilon}_{A}^{D^{\prime}}\left(v, v^{\prime}\right)$.

Since $s^{(q)} \neq \emptyset$, there are $\bar{u}, \bar{v} \in B$ such that $s_{B}^{(q)}(\bar{u}, \bar{v})$. Put

$$
x=(u, \bar{u}), \quad x^{\prime}=\left(u^{\prime}, \bar{u}\right), \quad y=(v, \bar{v}), \quad y^{\prime}=\left(v^{\prime}, \bar{v}\right) .
$$

Then $s^{(q)}(x, y)$ and $s^{(q)}\left(x^{\prime}, y^{\prime}\right)$, thus also $s(x, y)$ and $s\left(x^{\prime}, y^{\prime}\right)$. Hence assumption (\#) of the lemma implies $\varepsilon\left(x, x^{\prime}\right) \leftrightarrow \varepsilon\left(y, y^{\prime}\right)$. 
Suppose now that $\bar{\varepsilon}_{A}^{D}\left(u, u^{\prime}\right)$ is true; we have to deduce $\bar{\varepsilon}_{A}^{D^{\prime}}\left(v, v^{\prime}\right)$. Since $\bar{\varepsilon}_{B}^{D}(\bar{u}, \bar{u})$ holds obviously, $\bar{\varepsilon}^{D}\left(x, x^{\prime}\right)$ is true, and therefore $\varepsilon\left(x, x^{\prime}\right)$, by $\bar{\varepsilon}^{D} \subseteq \varepsilon$. We conclude that $\varepsilon\left(y, y^{\prime}\right)$. From $y, y^{\prime} \in \operatorname{rng}\left(s^{(q)}\right) \subseteq \bar{D}^{\prime}$ it follows that

$$
\varepsilon\left(y, y^{\prime}\right) \leftrightarrow \varepsilon\left((v, \bar{v}),\left(v^{\prime}, \bar{v}\right)\right) \leftrightarrow \varepsilon^{\bar{v}}\left(v, v^{\prime}\right) \leftrightarrow \bar{\varepsilon}_{A}^{D^{\prime}}\left(v, v^{\prime}\right),
$$

according to the definition of $\bar{\varepsilon}_{A}^{D^{\prime}}$ in 2.2 .

So we have deduced from $s_{A}^{(q)}(u, v) \wedge s_{A}^{(q)}\left(u^{\prime}, v^{\prime}\right)$ that $\bar{\varepsilon}_{A}^{D}\left(u, u^{\prime}\right) \rightarrow$ $\bar{\varepsilon}_{A}^{D^{\prime}}\left(v, v^{\prime}\right)$. The deduction of $\bar{\varepsilon}_{A}^{D^{\prime}}\left(v, v^{\prime}\right) \rightarrow \bar{\varepsilon}_{A}^{D}\left(u, u^{\prime}\right)$ is entirely symmetrical. So we have shown that $\overline{\bar{s}}_{A}^{(q)}$ is the graph of an injection. The proof for $\overline{\bar{s}}_{B}^{(q)}$ is analogous.

Denoting by $\widetilde{s}_{A}^{(q)}$ and $\widetilde{s}_{B}^{(q)}$ the injections whose graphs are $\overline{\bar{s}}_{A}^{(q)}$ and $\overline{\bar{s}}_{B}^{(q)}$, we define $\widetilde{s}^{(q)}=\widetilde{s}_{A}^{(q)} \times \widetilde{s}_{B}^{(q)}$. Then $\widetilde{s}^{(q)}$ is a local injection on $\mathbb{C}$ with $\operatorname{dom}\left(\widetilde{s}^{(q)}\right)$ $\subseteq D$ and $\operatorname{rng}\left(\widetilde{s}^{(q)}\right) \subseteq D^{\prime}$; moreover, $\widetilde{s}^{(q)}$ splits. One checks easily that $s^{(q)} / \bar{\varepsilon}$ is the graph of $\widetilde{s}^{(q)}$, whence $\widetilde{s}^{(q)}$ is definable and, for all $x, y \in \mathbb{C}$,

$$
\left(s^{(q)} / \bar{\varepsilon}\right)(x, y) \leftrightarrow \widetilde{s}^{(q)}(x)=y .
$$

It is now clear that if we put $\Gamma_{s}=\left\{\widetilde{s}^{(q)}: q=1, \ldots, r\right\}$ then assertion (1) of the lemma follows from $s / \bar{\varepsilon} \leftrightarrow \bigvee_{q=1}^{r}\left(s^{(q)} / \bar{\varepsilon}\right)$.

2.7. Lemma. If $\gamma \in \Gamma_{\varepsilon}$ and $\operatorname{dom}(\gamma), \operatorname{rng}(\gamma) \subseteq D$ for some $D \in \mathcal{D}_{\varepsilon}$ then none of the projections $\gamma_{A}, \gamma_{B}$ has a fixed point, unless $\gamma$ is the identity map on its domain.

Proof. We consider the proof of 2.6 for the case when $s$ is $\varepsilon$. Then $\gamma=\widetilde{\varepsilon}^{(q)}$ for some $q \in\{1, \ldots, r\}$, where $\varepsilon^{(q)}=\varepsilon_{A}^{(q)} \times \varepsilon_{B}^{(q)}$ and it may be assumed that $\varepsilon_{A}^{(q)}$ is $\bar{\varepsilon}_{A}^{D}$-invariant and $\varepsilon_{B}^{(q)}$ is $\bar{\varepsilon}_{B}^{D}$-invariant (see proof of Lemma 2.6). It follows that for all $(u, v),\left(u^{\prime}, v^{\prime}\right) \in \bar{D}$,

$$
\varepsilon_{A}^{(q)}\left(u, u^{\prime}\right) \leftrightarrow \gamma_{A}\left(u / \bar{\varepsilon}_{A}^{D}\right)=u^{\prime} / \bar{\varepsilon}_{A}^{D}, \quad \varepsilon_{B}^{(q)}\left(v, v^{\prime}\right) \leftrightarrow \gamma_{B}\left(v / \bar{\varepsilon}_{B}^{D}\right)=v^{\prime} / \bar{\varepsilon}_{B}^{D} .
$$

We claim that it will be enough to show that

$(\# \#) \quad\left(\varepsilon_{A}^{(q)}\left(u, u^{\prime}\right) \wedge \bar{\varepsilon}_{A}^{D}\left(u, u^{\prime}\right)\right) \rightarrow \varepsilon_{B}^{(q)}\left(v, v^{\prime}\right) \rightarrow \bar{\varepsilon}_{B}^{D}\left(v, v^{\prime}\right)$.

Indeed, this will prove that if $u / \bar{\varepsilon}_{A}^{D}$ is a fixed point for $\gamma_{A}$ then $\gamma_{B}$ is the identity map on its domain. Therefore $\gamma_{B}$ will have a fixed point, and by reversing the argument (interchanging $A$ with $B$ ) we deduce that also $\gamma_{A}$ is the identity map on its domain.

To prove (\#\#), assume that $\varepsilon_{A}^{(q)}\left(u, u^{\prime}\right)$ and $\bar{\varepsilon}_{A}^{D}\left(u, u^{\prime}\right)$ hold for some $u, u^{\prime} \in$ $\bar{D}_{A}$. Since $\varepsilon_{A}^{(q)}$ is $\bar{\varepsilon}_{A}^{D}$-invariant, it follows that $\varepsilon_{A}^{(q)}(u, u)$ holds. Suppose further that $\varepsilon_{B}^{(q)}\left(v, v^{\prime}\right)$ holds; we have to show that then also $\bar{\varepsilon}_{B}^{D}\left(v, v^{\prime}\right)$ is true. For this we put $x=(u, v)$ and $x^{\prime}=\left(u, v^{\prime}\right)$, so that

$$
\varepsilon^{(q)}\left(x, x^{\prime}\right) \leftrightarrow \varepsilon_{A}^{(q)}(u, u) \wedge \varepsilon_{B}^{(q)}\left(v, v^{\prime}\right),
$$


whence $\varepsilon^{(q)}\left(x, x^{\prime}\right)$ is true, and thus also $\varepsilon\left(x, x^{\prime}\right)$. But

$$
\varepsilon\left(x, x^{\prime}\right) \leftrightarrow \varepsilon\left((u, v),\left(u, v^{\prime}\right)\right) \leftrightarrow \varepsilon^{u}\left(v, v^{\prime}\right) \leftrightarrow \bar{\varepsilon}_{B}^{D}\left(v, v^{\prime}\right),
$$

because $u \in \bar{D}_{A}, v, v^{\prime} \in \bar{D}_{B}$. Thus $\bar{\varepsilon}_{B}^{D}\left(v, v^{\prime}\right)$ holds, and (\#\#) is proved.

2.8. Corollary. No $\gamma \in \Gamma_{\varepsilon}$ has a fixed point, unless $\gamma$ is the identity map on its domain.

Indeed, $\operatorname{dom}(\gamma)$ and $\operatorname{rng}(\gamma)$ are rectangles in $\mathbb{C}$, hence they are contained in some $D, D^{\prime} \in \mathcal{D}_{\varepsilon}$, so if $\gamma(x)=x$ for some $x$ then $D=D^{\prime}$ and Lemma 2.7 applies.

By Lemma 2.6, taken with $s=\varepsilon$, we obtain, for all $x, y \in \mathbb{C}$,

$$
\widetilde{\varepsilon}(x, y) \leftrightarrow \exists\left(\gamma \in \Gamma_{\varepsilon}\right)(\gamma(x)=y) .
$$

We shall presently deduce from this connection between $\widetilde{\varepsilon}$ and $\Gamma_{\varepsilon}$ a rather special partition $\mathcal{E}$ of $\mathbb{C}$. We shall describe this partition quite generally, for any set $\Delta$ (in particular, $\Delta=\mathbb{C}$ ) and a finite set $\Gamma$ of local injections on $\Delta$ (in particular, $\Gamma=\Gamma_{\varepsilon}$ ).

If $x \in \Delta$ and $\alpha \in \Gamma$, we shall say that $\alpha(x)$ is defined if $x \in \operatorname{dom}(\alpha)$. Saying that $\beta(\alpha(x))$ is defined will mean that $x \in \operatorname{dom}(\alpha), \beta \in \Gamma$ and $\alpha(x) \in$ $\operatorname{dom}(\beta)$. We extend this convention to longer composites like $\beta(\alpha(\kappa(x)))$.

2.9. Lemma. Let $\Delta$ be a set, let $\Gamma$ be a finite set of local injections on $\Delta$ and suppose that:

(I) No $\gamma \in \Gamma$ has a fixed point, unless $\gamma$ is the identity map on its domain.

(II) If $\alpha\left(x_{0}\right)$ is defined for some $x_{0} \in \Delta$ and $\alpha \in \Gamma$ then there is a $\beta \in \Gamma$ such that $\beta\left(\alpha\left(x_{0}\right)\right)=x_{0}$.

(III) If $\beta\left(\alpha\left(x_{0}\right)\right)$ is defined for some $x_{0} \in \Delta$ and $\alpha, \beta \in \Gamma$ then there exists a $\gamma \in \Gamma$ such that $\beta\left(\alpha\left(x_{0}\right)\right)=\gamma\left(x_{0}\right)$.

(IV) For every $x \in \Delta$ there is an $\alpha \in \Gamma$ such that $\alpha(x)=x$.

Then there exists a finite partition $\mathcal{E}$ of $\Delta$ such that for each $E \in \mathcal{E}$ and $\alpha \in \Gamma$ :

(i) $E \subseteq \operatorname{dom}(\alpha)$ or $E \cap \operatorname{dom}(\alpha)=\emptyset$,

(ii) if $\beta\left(\alpha\left(x_{0}\right)\right)$ is defined for some $\beta \in \Gamma$ and $x_{0} \in E$ then there is a $\gamma \in \Gamma$ such that $E \subseteq \operatorname{dom}(\gamma)$ and $\beta(\alpha(x))=\gamma(x)$ for all $x \in E$,

(iii) if $E \subseteq \operatorname{dom}(\alpha)$ then $\alpha(E) \in \mathcal{E}$.

Proof. We associate with each $x \in \Delta$ its $\Gamma$-character $\chi(x) \in \Gamma^{3}$ defined by

$$
\chi(x)=\left\{(\alpha, \beta, \gamma) \in \Gamma^{3}: \beta(\alpha(x))=\gamma(x)\right\} .
$$

Clearly, there are only finitely many distinct characters $\chi(x)$ for $x \in \Delta$. We define an equivalence relation $\cong_{\chi}$ on $\Delta$ by $x \cong_{\chi} y \leftrightarrow \chi(x)=\chi(y)$. Let 
$\mathcal{E}$ be the set of $\left(\cong_{\chi}\right)$-equivalence classes. Evidently $\mathcal{E}$ is a finite partition of $\Delta$, so it remains to verify that (i)-(iii) are true.

To prove (i), we have to show that if $\chi(x)=\chi(y)$ and $\alpha(x)$ is defined then $\alpha(y)$ is defined. Suppose $\alpha(x)$ is defined. Then, by (II), $\beta(\alpha(x))=x$ for some $\beta \in \Gamma$ and thus, by (III), $\beta(\alpha(x))=\gamma(x)$ for some $\gamma \in \Gamma$. Hence $(\alpha, \beta, \gamma) \in \chi(x)$, whence also $(\alpha, \beta, \gamma) \in \chi(y)$. Thus $\alpha(y)$ is defined.

Also (ii) has a simple proof. Indeed, by (III), there is a $\gamma \in \Gamma$ such that $\beta\left(\alpha\left(x_{0}\right)\right)=\gamma\left(x_{0}\right)$, so that $(\alpha, \beta, \gamma) \in \chi\left(x_{0}\right)$. If $x \in E$ then $\chi(x)=\chi\left(x_{0}\right)$, whence $(\alpha, \beta, \gamma) \in \chi(x)$ and $\beta(\alpha(x))=\gamma(x)$.

We claim that to prove (iii), it will be enough to show that if $\chi(x)=\chi(y)$ and both $\kappa(x)$ and $\kappa(y)$ are defined for some $\kappa \in \Gamma$ then $\chi(\kappa(x))=\chi(\kappa(y))$. This is equivalent to saying that $E \subseteq \operatorname{dom}(\kappa)$ implies $\kappa(E) \subseteq E^{\prime}$ for some $E^{\prime} \in \mathcal{E}$. To show that, under this assumption, $\kappa(E)=E^{\prime}$, consider any $x_{0} \in E$. Then, by (II), there is a $\delta \in \Gamma$ such that $\delta\left(\kappa\left(x_{0}\right)\right)=x_{0}$. Thus $\kappa\left(x_{0}\right) \in \operatorname{dom}(\delta)$ and $E^{\prime} \subseteq \operatorname{dom}(\delta)$, by (i).

Moreover, by (ii), there is a $\gamma \in \Gamma$ such that $\delta(\kappa(x))=\gamma(x)$ for all $x \in E$. Thus $\gamma\left(x_{0}\right)=x_{0}$, and so, by (I), $\gamma(x)=x$ for all $x \in \operatorname{dom}(\gamma)$. This means that for all $x \in E, \delta(\kappa(x))=x$. By the adopted assumption, $E^{\prime} \subseteq \operatorname{dom}(\delta)$ implies that $\delta\left(E^{\prime}\right) \subseteq E^{\prime \prime}$ for some $E^{\prime \prime} \in \mathcal{E}$. But then it follows from $\delta(\kappa(x))=$ $x$ on $E$ and $\kappa(E) \subseteq E^{\prime}$ that $E^{\prime \prime}$ meets $E$, whence $E^{\prime \prime}=E$. Suppose now that $\kappa(E)$ is a proper subset of $E^{\prime}$. Taking $y \in E^{\prime} \backslash \kappa(E)$, we have $\delta(y) \in E^{\prime \prime}=E$, say $\delta(y)=x \in E$. But also $\delta(\kappa(x))=x$, so that $\delta(y)=\delta(\kappa(x))$. Since $y \notin$ $\kappa(E)$, we reached a contradiction with the injectivity of $\delta$. So $\kappa(E)=E^{\prime} \in \mathcal{E}$.

It still remains to show that if $\chi(x)=\chi(y)$ and $\kappa(x), \kappa(y)$ are defined then $\chi(\kappa(x))=\chi(\kappa(y))$. Obviously, by symmetry, showing $\chi(\kappa(x)) \subseteq$ $\chi(\kappa(y))$ will do. So, suppose that $(\alpha, \beta, \gamma) \in \chi(\kappa(x))$. This means that

$$
\beta(\alpha(\kappa(x)))=\gamma(\kappa(x)),
$$

and the conclusion to be reached is the same equality with $x$ replaced by $y$. By (III), $\alpha(\kappa(x))=\lambda(x)$ and $\gamma(\kappa(x))=\varrho(x)$ for some $\lambda, \varrho \in \Gamma$. Thus $(\S \S)$ becomes $\beta(\lambda(x))=\varrho(x)$; moreover, in the last three equalities, we can replace everywhere $x$ by $y$, in view of $\chi(x)=\chi(y)$, thus getting

$$
\alpha(\kappa(y))=\lambda(y), \quad \gamma(\kappa(y))=\varrho(y), \quad \beta(\lambda(y))=\varrho(y) .
$$

These three equalities imply that

$$
\beta(\alpha(\kappa(y)))=\beta(\lambda(y))=\varrho(y)=\gamma(\kappa(y)) .
$$

2.10. Corollary. There exists a finite partition $\mathcal{E}$ of $\mathbb{C}$ such that every $E \in \mathcal{E}$ is definable and contained in some $D \in \mathcal{D}_{\varepsilon}$, and moreover, for every $\alpha \in \Gamma_{\varepsilon}$ and $E \in \mathcal{E}$ all the assertions (i)-(iii) of Lemma 2.9 hold.

Proof. It follows from Corollary 2.8 and (§) that the assumptions (I)(IV) of Lemma 2.9 hold if we put $\Delta=\mathbb{C}$ and $\Gamma=\Gamma_{\varepsilon}$. Applying Lemma 2.9, we get the partition $\mathcal{E}$ of $\mathbb{C}$ satisfying (i)-(iii) for every $E \in \mathcal{E}$ and $\alpha \in \Gamma_{\varepsilon}$. 
By (i) and (IV) of that lemma, for each $E \in \mathcal{E}$ there is an $\alpha \in \Gamma_{\varepsilon}$ such that $E \subseteq \operatorname{dom}(\alpha)$. But $\operatorname{dom}(\alpha)$ is a rectangle in $\mathbb{C}$, thus $E \subseteq D$ for some $D \in \mathcal{D}_{\varepsilon}$. The definability of $E$ (i.e., of $E \cdot \bar{\varepsilon}^{D}$, in the notation of 2.2) is not hard to deduce from the definition of $\mathcal{E}$.

2.11. Corollary. Given $E \in \mathcal{E}$, let $G_{E}$ denote the set of the restrictions $\alpha \uparrow E$ of those $\alpha \in \Gamma_{\varepsilon}$ for which $\alpha(E)=E$. Then $G_{E}$ is a group of bijections $E \rightarrow E$. Moreover, every non-empty intersection of any $\widetilde{\varepsilon}$-equivalence class with $E$ is a $G_{E}$-orbit.

Proof. Let us show the existence of an inverse for each $g \in G_{E}$. So let $g \in G_{E}$, i.e., $g=\alpha \mid E$, where $\alpha \in \Gamma_{\varepsilon}$ and $\alpha(E)=E$. Choosing any $x_{0} \in E$, we have a $\beta \in \Gamma_{\varepsilon}$ such that $\beta\left(\alpha\left(x_{0}\right)\right)=x_{0}$, and thus $\beta(\alpha(x))=\gamma(x)$ for some $\gamma \in \Gamma_{\varepsilon}$ and all $x \in E$ (by (II) and (ii) of Lemma 2.9). So $E \subseteq \operatorname{dom}(\gamma)$ and since also $\gamma\left(x_{0}\right)=x_{0}$, we get $\gamma(x)=x$ on $E$, by (I). Consequently, $\beta(\alpha(x))=x$ on $E$, which implies $\beta(E)=E$. The inverse of $g$ is thus $\beta\lceil E$. The fact that the composite of two bijections $g, h \in G_{E}$ is also in $G_{E}$ follows from (ii) of Lemma 2.9.

So $G_{E}$ is a group. The asserted connection between the $\widetilde{\varepsilon}$-equivalence classes and $G_{E}$-orbits follows from $(\S)$ and from the fact that if $\alpha(x) \in E$ for some $x \in E$ and $\alpha \in \Gamma_{\varepsilon}$ then $\alpha(E)=E$, by (i) and (iii) of Lemma 2.9.

If a group $G$ acts on a set $S$ (as a group of bijections) and $\mathcal{M}$ is a family of subsets of $S$, we shall say that $\mathcal{M}$ is $G$-invariant if $g M \in \mathcal{M}$ for every $M \in \mathcal{M}$.

2.12. Lemma. For every $E \in \mathcal{E}$, there exists a finite $G_{E}$-invariant partition $\mathcal{R}_{E}$ of $E$ such that each $R \in \mathcal{R}_{E}$ is a definable rectangle in $\mathbb{C}$.

Proof. Let $E \in \mathcal{E}$ be fixed. Let us show first that $E$ is the union of a finite family $\mathbb{Q}$ of definable rectangles in $\mathbb{C}$. If $E \subseteq D$ with $D \in \mathcal{D}_{\varepsilon}$ (see Corollary 2.10), let us denote by $E_{A}$ and $E_{B}$ the projections of $E$ on $D_{A}$ and $D_{B}$, so that $E \subseteq E_{A} \times E_{B}$. Since $E \cdot \bar{\varepsilon}^{D} \subseteq C$ is definable, $E \cdot \bar{\varepsilon}^{D}$ is a finite union of definable rectangles. If $P=P_{A} \times P_{B}$ is any of these rectangles contained in $E \cdot \bar{\varepsilon}^{D}$ then we can assume that $P$ is $\bar{\varepsilon}^{D}$-invariant, for otherwise $P$ can be replaced by $\bar{P}=\bar{P}_{A} \times \bar{P}_{B}$, where for each $u \in \bar{D}_{A}$ and $v \in \bar{D}_{B}$,

$$
u \in \bar{P}_{A} \leftrightarrow\left(\exists u^{\prime} \in P_{A}\right)\left(\bar{\varepsilon}_{A}^{D}\left(u, u^{\prime}\right)\right), \quad v \in \bar{P}_{B} \leftrightarrow\left(\exists v^{\prime} \in P_{B}\right)\left(\bar{\varepsilon}_{B}^{D}\left(v, v^{\prime}\right)\right) .
$$

However, an $\bar{\varepsilon}^{D}$-invariant, definable rectangle $P$ in $E \cdot \bar{\varepsilon}^{D}$ is of the form $Q \cdot \bar{\varepsilon}^{D}$, where $Q$ is a definable rectangle contained in $E$. It follows that $E=\bigcup \mathbb{Q}$ for a finite family $\mathbb{Q}$ of definable rectangles in $\mathbb{C}$.

We now put

$$
\mathbb{Q}_{A}=\left\{Q_{A} \subseteq E_{A}: Q_{A} \times Q_{B} \in \mathbb{Q}\right\}, \quad \mathbb{Q}_{B}=\left\{Q_{B} \subseteq E_{B}: Q_{A} \times Q_{B} \in \mathbb{Q}\right\} .
$$

So $\mathbb{Q}_{A}$ is a finite family of sets such that for every $Q_{A} \in \mathbb{Q}_{A}$ the set $Q_{A} \cdot \bar{\varepsilon}_{A}^{D}$ is $\mathfrak{A}$-definable and $E_{A}=\bigcup \mathbb{Q}_{A}$. Similarly $E_{B}=\bigcup \mathbb{Q}_{B}$. For every $g \in G_{E}$, 
we have $g=g_{A} \times g_{B}$, where $g_{A}$ permutes $E_{A}$, and $g_{B}$ permutes $E_{B}$ (see Lemma 2.6(2), with $s=\varepsilon)$. Clearly, the finite family of $\mathfrak{A}$-definable sets

$$
G_{A}\left(\mathbb{Q}_{A}\right)=\left\{g_{A} Q_{A} \subseteq E_{A}: g \in G_{E}, Q_{A} \in \mathbb{Q}_{A}\right\}
$$

is $G_{E}$-invariant. So, if $\mathcal{R}_{A}$ is the set of atoms of the (finite) Boolean algebra of subsets of $E_{A}$ generated by $G_{A}\left(\mathbb{Q}_{A}\right)$ then $\mathcal{R}_{A}$ is a $G_{E}$-invariant partition of $E_{A}$ such that for every $R_{A} \in \mathcal{R}_{A}$ the set $R_{A} \cdot \bar{\varepsilon}_{A}^{D}$ is $\mathfrak{A}$-definable. We define similarly the $G_{E}$-invariant partition $\mathcal{R}_{B}$ of $E_{B}$. The required partition $\mathcal{R}_{E}$ of $E$ into finitely many definable rectangles in $\mathbb{C}$ is now given by

$$
\mathcal{R}_{E}=\left\{R_{A} \times R_{B}: R_{A} \in \mathcal{R}_{A}, R_{B} \in \mathcal{R}_{B} \text { and } R_{A} \times R_{B} \subseteq E\right\} .
$$

Clearly the $G_{E^{-}}$-invariance of $\mathcal{R}_{A}$ and $\mathcal{R}_{B}$ implies that $\mathcal{R}_{E}$ is also $G_{E^{-}}$ invariant.

2.13. Corollary. Given $E \in \mathcal{E}$ and $R \in \mathcal{R}_{E}$, the restrictions $g \backslash R$ of those $g \in G_{E}$ for which $g R=R$ form a group $G_{R}$ of bijections $R \rightarrow R$.

2.14. Pro of of Theorem 2.3. Let $\overline{\mathcal{E}}$ denote a subfamily of $\mathcal{E}$ such that every $\widetilde{\varepsilon}$-equivalence class meets exactly one $E \in \mathcal{E}$. To show the existence of $\overline{\mathcal{E}}$, we consider the binary relation $\equiv$ on $\mathcal{E}$ defined by

$$
E_{1} \equiv E_{2} \leftrightarrow \exists\left(\alpha \in \Gamma_{\varepsilon}\right)\left(\alpha\left(E_{1}\right)=E_{2}\right) .
$$

We claim that $\equiv$ is an equivalence relation. To prove this, we shall use repeatedly the properties (I)-(iii) listed in Lemma 2.9 (for $\Delta=\mathbb{C}, \Gamma=$ $\Gamma_{\varepsilon}$ ), without mentioning them explicitly. The required verifications may be indicated thus:

Reflexivity: Suppose $E \in \mathcal{E}$. Taking any $x_{0} \in E$, we find an $\alpha \in \Gamma_{\varepsilon}$ such that $\alpha\left(x_{0}\right)=x_{0}$. Thus $\alpha(E)=E$ (by (i), (ii)).

Symmetry: Suppose $\alpha\left(E_{1}\right)=E_{2}$. Taking any $x_{0} \in E_{1}$, we find a $\beta \in \Gamma_{\varepsilon}$ such that $\beta\left(\alpha\left(x_{0}\right)\right)=x_{0}$, whence $E_{2} \subseteq \operatorname{dom}(\beta)$ and $\beta(\alpha(x))=x$ on $E_{1}$ and thus $\beta\left(E_{2}\right)=E_{1}$.

Transitivity: Suppose that $\alpha\left(E_{1}\right)=E_{2}$ and $\beta\left(E_{2}\right)=E_{3}$ for $E_{1}, E_{2}, E_{3} \in$ $\mathcal{E}$ and $\alpha, \beta \in \Gamma_{\varepsilon}$. Let $x_{0} \in E_{1}$; thus $\beta\left(\alpha\left(x_{0}\right)\right)$ is defined, whence there is a $\gamma \in \Gamma_{\varepsilon}$ such that $\beta(\alpha(x))=\gamma(x)$ on $E_{1}$ and thus $\gamma\left(E_{1}\right)=E_{3}$.

Let $\overline{\mathcal{E}} \subseteq \mathcal{E}$ be a set of representatives of the (三)-equivalence classes in $\mathcal{E}$. Then for every $x \in \mathbb{C}$ there is exactly one $E \in \overline{\mathcal{E}}$ such that $\gamma(x) \in E$ for some $\gamma \in \Gamma_{\varepsilon}$. So, in view of (§), every $\widetilde{\varepsilon}$-equivalence class intersects exactly one $E \in \overline{\mathcal{E}}$.

Next, for every $E \in \overline{\mathcal{E}}$, let $\overline{\mathcal{R}}_{E}$ denote a subfamily of $\mathcal{R}_{E}$ (see Lemma 2.12) such that each $G_{E}$-orbit intersects exactly one $R \in \overline{\mathcal{R}}_{E}$. Such a subfamily is obtained by considering on $\mathcal{R}_{E}$ the equivalence relation

$$
R_{1} \cong R_{2} \leftrightarrow \exists\left(g \in G_{E}\right)\left(g R_{1}=R_{2}\right),
$$




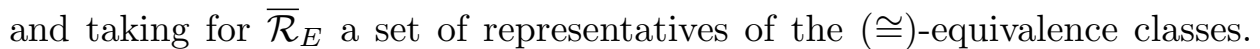
Then for each $x \in E$, there is exactly one $R \in \overline{\mathcal{R}}_{E}$ such that $g x \in R$ for some $g \in G_{E}$.

We now define $\mathcal{R}=\bigcup\left\{\overline{\mathcal{R}}_{E}: E \in \overline{\mathcal{E}}\right\}$. It remains to check (1) and (2) of Theorem 2.3. Clearly, (1) is a consequence of the following two facts:

(a) every $\widetilde{\varepsilon}$-equivalence class intersects exactly one $E \subseteq \overline{\mathcal{E}}$ and their intersection is a $G_{E}$-orbit (Corollary 2.11),

(b) every $G_{E}$-orbit intersects exactly one $R \in \overline{\mathcal{R}}_{E}$, and their intersection is a $G_{R}$-orbit (Lemma 2.12 and Corollary 2.13).

To check (2), consider any $R \in \mathcal{R}$ and $D \in \mathcal{D}_{\varepsilon}$ such that $R \subseteq D$. Suppose $g \in G_{R}$, i.e., $g$ is the restriction to $R$ of some definable and splitting local injection $\gamma \in \Gamma_{\varepsilon}$ on $\mathbb{C}$ such that $\gamma(R)=R$. So $g$ is definable and it splits; in fact, $g=g_{A} \times g_{B}$, where $g_{A}$ and $g_{B}$ are the restrictions of $\gamma_{A}$ to $R_{A}$ and of $\gamma_{B}$ to $R_{B}$. Thus $g_{A}$ and $g_{B}$ are bijective. Since $\operatorname{dom}(\gamma), \operatorname{rng}(\gamma) \subseteq D$, Lemma 2.7 can be applied and we conclude that none of the projections $\gamma_{A}, \gamma_{B}$ has a fixed point, unless $\gamma$ is the identity map on its domain. Suppose $g$ is not the identity map on $R$. Then $\gamma$ is not the identity map on its domain, and hence $\gamma_{A}, \gamma_{B}$ have no fixed points. It follows that $g_{A}$ and $g_{B}$ have no fixed points.

\section{Partitions for the graph of an injection}

3.1. Suppose $(\varepsilon, r)$ is a pair interpreting $\mathbf{T}$ (see 1.1). Let us denote by $\mathbb{R} \subseteq$ $\mathbb{C}$ the disjoint union $\bigcup \mathcal{R}$, where $\mathcal{R}$ is the family of rectangles in Theorem 2.3. Let further $\widetilde{r}$ denote the binary relation $r / \bar{\varepsilon}$ on $\mathbb{C}$ (see 2.2). In this section we shall introduce two partitions $\Pi$ and $\Omega$ of $\mathbb{R}$ which are induced by $\widetilde{r}$.

Since $r$ satisfies the assumption of Lemma 2.6 (with $s$ replaced by $r$ ), there corresponds to this relation the finite set $\Gamma_{r}$ of local injections on $\mathbb{C}$, given by that lemma. Let $\Gamma_{r}^{\mathcal{R}}$ be the set of restrictions of all $\gamma \in \Gamma_{r}$ to the rectangles of $\mathcal{R}$. More precisely, consider for every $R, R^{\prime} \in \mathcal{R}$ and $\gamma \in \Gamma_{r}$ the set $\left\{x \in R: \gamma(x) \in R^{\prime}\right\}$, and if this set is non-empty, take it to be the domain of the local injection $\varphi$ from $R$ to $R^{\prime}$, given by $\varphi(x)=\gamma(x) . \Gamma_{r}^{\mathcal{R}}$ is defined to be the (finite) set of all local injections on $\mathbb{R}$, obtained in this way. It is clear that each $\varphi \in \Gamma_{r}^{\mathcal{R}}$ is definable and that $\varphi$ splits. Moreover, by Lemma 2.6, we have, for every $x, y \in \mathbb{R}$,

$$
\widetilde{r}(x, y) \leftrightarrow \exists\left(\varphi \in \Gamma_{r}^{\mathcal{R}}\right)(\varphi(x)=y) .
$$

R e m a r k. Applying Theorem 2.3, we conclude from (o) that $\mathbb{R}$ is covered by the domains of all $\varphi \in \Gamma_{r}^{\mathcal{R}}$.

We shall denote by $\mathbb{G}$ the disjoint union $\bigcup\left\{G_{R}: R \in \mathcal{R}\right\}$ and call every $G_{R}$-orbit in $R \in \mathcal{R}$ a $\mathbb{G}$-orbit. By Theorem 2.3 , the $\mathbb{G}$-orbits are the intersections of the $\widetilde{\varepsilon}$-equivalence classes with $\mathbb{R}$. 
A partition $\Pi$ of $\mathbb{R}$ will be called $\mathbb{G}$-invariant if $\Pi=\bigcup\left\{\Pi_{R}: R \in \mathcal{R}\right\}$, where each $\Pi_{R}$ is a $G_{R}$-invariant partition of $R$. For any set $S \subseteq R(R \in \mathcal{R})$, $G_{S}$ will denote the stabilizer of $S$, i.e., the subgroup of $G_{R}$ composed of those $g: R \rightarrow R$ for which $g S=S$. The number of elements of $G_{S}$ will be denoted by $\left|G_{S}\right|$.

3.2. TheOREM. There exist finite $\mathbb{G}$-invariant partitions $\Pi$ and $\Omega$ of $\mathbb{R}$ into definable sets such that, for every $\varphi \in \Gamma_{r}^{\mathcal{R}}$ :

(1) $\operatorname{dom}(\varphi)$ is partitioned by sets belonging to $\Pi$,

(2) $\operatorname{rng}(\varphi)$ is partitioned by sets belonging to $\Omega$,

(3) if $U \subseteq \operatorname{dom}(\varphi)$ and $U \in \Pi$ then $\varphi(U) \in \Omega$ and $\left|G_{U}\right|=\left|G_{\varphi(U)}\right|$; moreover, there is a $V_{*} \in \Omega$ such that $g V_{*}$ is disjoint from each $\varphi(U)$, for all $g \in \mathbb{G}$ and $U \in \Pi, \varphi \in \Gamma_{r}^{\mathcal{R}}$.

3.3. To begin the proof, let us establish two properties $(*)$ and $(* *)$ of $\mathbb{G}$ and $\Gamma_{r}^{\mathcal{R}}$ which will underlie the construction of $\Pi$ and $\Omega$.

The $\varepsilon$-invariance of $r$ implies that $\widetilde{r}$ is $\widetilde{\varepsilon}$-invariant, that is,

$$
\widetilde{\varepsilon}\left(x, x^{\prime}\right) \wedge \widetilde{\varepsilon}\left(y, y^{\prime}\right) \rightarrow\left(\widetilde{r}(x, y) \leftrightarrow \widetilde{r}\left(x^{\prime}, y^{\prime}\right)\right),
$$

for all $x, x^{\prime}, y, y^{\prime} \in \mathbb{C}$. Hence, by (o) and Theorem 2.3, we have, for all $x, x^{\prime}, y, y^{\prime} \in \mathbb{R}:$

(*) If $x$ and $x^{\prime}$ are on the same $\mathbb{G}$-orbit and $y$ and $y^{\prime}$ are on the same $\mathbb{G}$-orbit then there is a $\varphi \in \Gamma_{r}^{\mathcal{R}}$ satisfying $\varphi(x)=y$ iff there is a $\varphi^{\prime} \in \Gamma_{r}^{\mathcal{R}}$ satisfying $\varphi^{\prime}\left(x^{\prime}\right)=y^{\prime}$.

Next, let us observe that $\widetilde{r} / \widetilde{\varepsilon}$ is the graph of an injection of $\mathbb{C} / \widetilde{\varepsilon}$ into itself. (This can be seen by identifying in the obvious way $\mathbb{C} / \widetilde{\varepsilon}=(C / \bar{\varepsilon}) /(\varepsilon / \bar{\varepsilon})$ with $C / \varepsilon$ and $\widetilde{r} / \widetilde{\varepsilon}=(r / \bar{\varepsilon}) /(\varepsilon / \bar{\varepsilon})$ with $r / \varepsilon$.) Thus

$$
\widetilde{r}(x, y) \wedge \widetilde{r}\left(x^{\prime}, y^{\prime}\right) \rightarrow\left(\widetilde{\varepsilon}\left(x, x^{\prime}\right) \leftrightarrow \widetilde{\varepsilon}\left(y, y^{\prime}\right)\right)
$$

for all $x, x^{\prime} y, y^{\prime} \in \mathbb{C}$. Hence, by (o) and Theorem 2.3, we have, for all $x, x^{\prime}, y, y^{\prime} \in \mathbb{R}:$

$(* *) \quad$ If $\varphi(x)=y$ and $\varphi^{\prime}\left(x^{\prime}\right)=y^{\prime}$ for some $\varphi, \varphi^{\prime} \in \Gamma_{r}^{\mathcal{R}}$ then $x$ and $x^{\prime}$ are on the same $\mathbb{G}$-orbit iff $y$ and $y^{\prime}$ are on the same $\mathbb{G}$-orbit.

3.4. The definitions of $\Pi$ and $\Omega$ are now as follows. Let us call a quadruple $\left(\varphi, \varphi^{\prime}, g, g^{\prime}\right)$, where $\varphi, \varphi^{\prime} \in \Gamma_{r}^{\mathcal{R}}, g, g^{\prime} \in \mathbb{G}$, a diagram. We shall say that this diagram commutes on $x \in \mathbb{R}$ if $g^{\prime} \varphi(x)=\varphi^{\prime}(g x)$. Given $x \in \mathbb{R}$, let $\operatorname{rt}(x)$ denote the (finite) set of all diagrams which commute on $x$. Let us say that $x, y \in \mathbb{R}$ are right equivalent iff $\operatorname{rt}(x)=\operatorname{rt}(y)$. We define $\Pi$ to be the set of equivalence classes for this relation of right equivalence on $\mathbb{R}$. Evidently $\Pi$ is a finite partition of $\mathbb{R}$ and each $U \in \Pi$ is definable.

To establish assertion (1) of the theorem, consider any $x \in U \cap \operatorname{dom}(\varphi)$, where $U \in \Pi$ and $\varphi \in \Gamma_{r}^{\mathcal{R}}$. If $R, R^{\prime} \in \mathcal{R}$ are the sets containing $\operatorname{dom}(\varphi)$ 
and $\operatorname{rng}(\varphi)$, respectively, then $\left(\varphi, \varphi, 1_{R}, 1_{R^{\prime}}\right) \in \operatorname{rt}(x)$, where $1_{R}$ and $1_{R^{\prime}}$ are the identities of the groups $G_{R}$ and $G_{R^{\prime}}$. Then $\left(\varphi, \varphi, 1_{R}, 1_{R^{\prime}}\right) \in \operatorname{rt}(y)$ for all $y \in U$, whence $U \subseteq \operatorname{dom}(\varphi)$. This proves assertion (1).

To define $\Omega$, let us denote for all $x \in \mathbb{R}$ by $\operatorname{lt}(x)$ the set of all diagrams $\left(\varphi, \varphi^{\prime}, g, g^{\prime}\right)$ such that $x \in \operatorname{rng}(\varphi)$ and the diagram commutes on $\varphi^{-1}(x)$ (i.e., $\left.\left(\varphi, \varphi^{\prime}, g, g^{\prime}\right) \in \operatorname{rt}\left(\varphi^{-1}(x)\right)\right)$.

We put $V_{0}=\{x \in \mathbb{R}: \operatorname{lt}(x)=\emptyset\}$. Let us show that $V_{0} \neq \emptyset$ and that $x \in V_{0}$ iff $x$ does not belong to any $\operatorname{rng}(\varphi)$, where $\varphi \in \Gamma_{r}^{\mathcal{R}}$. Indeed, if $x \in \operatorname{rng}(\varphi)$, where $\varphi \in \Gamma_{r}^{\mathcal{R}}$, then for the sets $R, R^{\prime} \in \mathcal{R}$ such that $\operatorname{dom}(\varphi) \subseteq R$ and $\operatorname{rng}(\varphi) \subseteq R^{\prime}$, one has $\left(\varphi, \varphi, 1_{R}, 1_{R^{\prime}}\right) \in \operatorname{lt}(x)$, so $\operatorname{lt}(x) \neq \emptyset$. Conversely, if $\operatorname{lt}(x) \neq \emptyset$ then clearly $x \in \operatorname{rng}(\varphi)$ for some $\varphi \in \Gamma_{r}^{\mathcal{R}}$. Now, to prove that $V_{0} \neq \emptyset$, note that $\widetilde{r} / \widetilde{\varepsilon}$ is the graph of a non-surjective map of $\mathbb{C} / \widetilde{\varepsilon}$ into itself. Thus $\operatorname{rng}(\widetilde{r}) \neq \mathbb{C}$, and so it follows from Theorem 2.3 and (o) that there is an $x_{*} \in \mathbb{R}$ which does not belong to any $\operatorname{rng}(\varphi)$, where $\varphi \in \Gamma_{r}^{\mathcal{R}}$, i.e., $x_{*} \in V_{0}$, whence $V_{0} \neq \emptyset$.

Let us now call two elements $x, y \in \mathbb{R}$ left equivalent if

$$
(\operatorname{lt}(x)=\operatorname{lt}(y) \neq \emptyset) \vee \exists(R \in \mathcal{R})(x, y \in R \wedge \operatorname{lt}(x)=\operatorname{lt}(y)=\emptyset) .
$$

We denote by $\Omega$ the set of equivalence classes for this relation of left equivalence on $\mathbb{R}$. Then each non-empty intersection $V_{0} \cap R(R \in \mathcal{R})$ belongs to $\Omega$, and since $V_{0} \neq \emptyset$, at least one such set, say $V_{*}=V_{0} \cap R$, is non-empty. From the fact that $V_{*} \cap \operatorname{rng}(\varphi)=\emptyset$ for all $\varphi \in \Gamma_{r}^{\mathcal{R}}$ and from $(*)$ (taken for $x=x^{\prime}$ ) it follows that $g V_{*} \cap \operatorname{rng}(\varphi)=\emptyset$ for all $g \in \mathbb{G}$ and $\varphi \in \Gamma_{r}^{\mathcal{R}}$. This establishes the last assertion of the theorem.

We next claim that if $V \in \Omega, \varphi \in \Gamma_{r}^{\mathcal{R}}$ and $V \cap \operatorname{rng}(\varphi) \neq \emptyset$ then $V \subseteq \operatorname{rng}(\varphi)$. Indeed, if $\varphi(x) \in V$, where $x \in R$ and $\varphi(x) \in R^{\prime}$ with $R, R^{\prime} \in \mathcal{R}$, then $\left(\varphi, \varphi, 1_{R}, 1_{R^{\prime}}\right) \in \operatorname{lt}(x)=\operatorname{lt}(y)$ for all $y \in V$, whence $y \in \operatorname{rng}(\varphi)$ for all $y \in V$. Thus assertion (2) is proved.

To prove the $\mathbb{G}$-invariance of the partitions $\Pi, \Omega$ and property (3), we need Lemma 3.5 below. Let us call two diagrams of the form $\left(\varphi, \varphi^{\prime}, g_{1}, g_{2}\right)$, $\left(\varphi^{\prime}, \varphi^{\prime \prime}, g^{\prime}, g^{\prime \prime}\right)$ stacked. Such diagrams may be imagined as placed one above the other. Accordingly, we shall refer to $\left(\varphi, \varphi^{\prime}, g_{1}, g_{2}\right)$ as the upper and to $\left(\varphi^{\prime}, \varphi^{\prime \prime}, g^{\prime}, g^{\prime \prime}\right)$ as the lower diagram. The diagram $\left(\varphi, \varphi^{\prime \prime}, g^{\prime} g_{1}, g^{\prime \prime} g_{2}\right)$ will be called outer (see the diagram below).

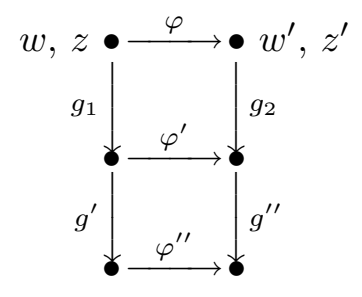


3.5. Lemma. Consider the two stacked diagrams above and let $w, z, w^{\prime}, z^{\prime}$ $\in \mathbb{R}$. Then:

(a) If $\operatorname{rt}(w)=\operatorname{rt}(z)$, the upper diagram belongs to $\operatorname{rt}(w)$ and the lower diagram belongs to $\operatorname{rt}\left(g_{1} w\right)$, then the lower diagram belongs to $\operatorname{rt}\left(g_{1} z\right)$.

(b) If $\operatorname{lt}\left(w^{\prime}\right)=\operatorname{lt}\left(z^{\prime}\right)$, the upper diagram belongs to $\operatorname{lt}\left(w^{\prime}\right)$ and the lower diagram belongs to $\operatorname{lt}\left(g_{2} w^{\prime}\right)$, then the lower diagram belongs to $\operatorname{lt}\left(g_{2} z^{\prime}\right)$.

Proof. (a) Under the stated assumptions, the outer diagram belongs to $\operatorname{rt}(w)$, hence to $\operatorname{rt}(z)$. But also the upper diagram belongs to $\operatorname{rt}(z)$, and therefore the lower diagram belongs to $\operatorname{rt}\left(g_{1} z\right)$.

(b) Put $w=\varphi^{-1}\left(w^{\prime}\right), z=\varphi^{-1}\left(z^{\prime}\right)$ and use (a).

3.6. Lemma. $\Pi$ is $\mathbb{G}$-invariant.

Proof. Before speaking about the $\mathbb{G}$-invariance of $\Pi$, we must check that every $R \in \mathcal{R}$ is partitioned by sets belonging to $\Pi$. Now, this is indeed the case, because each $R \in \mathcal{R}$ is a union of the domains of various $\varphi \in \Gamma_{r}^{\mathcal{R}}$, and we have already established (1). Thus it will be enough to show that for any $x, y \in R \in \mathcal{R}$ and $g_{1} \in G_{R}$,

$$
\operatorname{rt}(x)=\operatorname{rt}(y) \rightarrow \operatorname{rt}\left(g_{1} x\right)=\operatorname{rt}\left(g_{1} y\right)
$$

(then the reverse implication follows by applying $g_{1}^{-1}$ ). So let $\operatorname{rt}(x)=\operatorname{rt}(y)$. By symmetry, it will suffice to check that $\operatorname{rt}\left(g_{1} x\right) \subseteq \operatorname{rt}\left(g_{1} y\right)$. Assume therefore that $\left(\varphi^{\prime}, \varphi^{\prime \prime}, g^{\prime}, g^{\prime \prime}\right) \in \operatorname{rt}\left(g_{1} x\right)$. Let $\varphi \in \Gamma_{r}^{\mathcal{R}}$ be such that $x \in \operatorname{dom}(\varphi)$ (see remark after $(\circ))$. Since $g_{1} x \in \operatorname{dom}\left(\varphi^{\prime}\right)$, it follows from $(* *)$ that $\varphi(x)$ and $\varphi^{\prime}\left(g_{1} x\right)$ are on the same $\mathbb{G}$-orbit, i.e., $\left(\varphi, \varphi^{\prime}, g_{1}, g_{2}\right) \in \operatorname{rt}(x)$ for some $g_{2} \in \mathbb{G}$. We obtained two stacked diagrams, the upper one being $\left(\varphi, \varphi^{\prime}, g_{1}, g_{2}\right) \in$ $\operatorname{rt}(x)$, and the lower one $\left(\varphi^{\prime}, \varphi^{\prime \prime}, g^{\prime}, g^{\prime \prime}\right) \in \operatorname{rt}\left(g_{1} x\right)$. By Lemma 3.5(a), the lower diagram belongs to $\operatorname{rt}\left(g_{1} y\right)$.

3.7. Lemma. $\Omega$ is $\mathbb{G}$-invariant.

Pr o of. To show that each $R \in \mathcal{R}$ is partitioned by sets belonging to $\Omega$, it will suffice to check that each $V \in \Omega$ is contained in some $R \in \mathcal{R}$. This is indeed the case when $V \cap \operatorname{rng}(\varphi) \neq \emptyset$ for some $\varphi \in \Gamma_{r}^{\mathcal{R}}$. Then $V \subseteq \operatorname{rng}(\varphi)$ by assertion (2) and thus $V \subseteq R$ for the set $R \in \mathcal{R}$ containing $\operatorname{rng}(\varphi)$. If $V \cap \operatorname{rng}(\varphi)=\emptyset$ for all $\varphi \in \Gamma_{r}^{\overline{\mathcal{R}}}$ then $V=V_{0} \cap R$ for some $R \in \mathcal{R}$, by the definition of $\Omega$ (i.e., of left equivalence).

To prove the $\mathbb{G}$-invariance of $\Omega$, it will suffice to show that for any $x, y \in R \in \mathcal{R}$ and $g_{2} \in G_{R}$,

$$
\operatorname{lt}(x)=\operatorname{lt}(y) \rightarrow \operatorname{lt}\left(g_{2} x\right) \subseteq \operatorname{lt}\left(g_{2} y\right) .
$$

The proof is again an application of Lemma 3.5. Suppose that $\operatorname{lt}(x)=\operatorname{lt}(y)$ and $\left(\varphi^{\prime}, \varphi^{\prime \prime}, g^{\prime}, g^{\prime \prime}\right) \in \operatorname{lt}\left(g_{2} x\right)$. Then $\varphi^{\prime}\left(x^{\prime}\right)=g_{2} x$ for some $x^{\prime} \in R^{\prime} \in \mathcal{R}$. Since $x$ and $g_{2} x$ are on the same $\mathbb{G}$-orbit, an application of $(*)$ yields a map 
$\varphi \in \Gamma_{r}^{\mathcal{R}}$ such that $\varphi\left(x^{\prime}\right)=x$. This implies $\left(\varphi, \varphi^{\prime}, 1_{R^{\prime}}, g_{2}\right) \in \operatorname{lt}(x)$. We again obtained two stacked diagrams

$$
\left(\varphi, \varphi^{\prime}, 1_{R^{\prime}}, g_{2}\right) \in \operatorname{lt}(x), \quad\left(\varphi^{\prime}, \varphi^{\prime \prime}, g^{\prime}, g^{\prime \prime}\right) \in \operatorname{lt}\left(g_{2} x\right) .
$$

By Lemma 3.5(b), the second diagram belongs to $\operatorname{lt}\left(g_{2} y\right)$.

3.8. Lemma. If $U \in \Pi$ and $U \subseteq \operatorname{dom}(\varphi)$, where $\varphi \in \Gamma_{r}^{\mathcal{R}}$, then $\varphi(U) \in \Omega$.

Proof. Since (1) and (2) are already established, the lemma will follow if we show that for $x, y \in \mathbb{R}$ and $\varphi \in \Gamma_{r}^{\mathcal{R}}$,

$$
\operatorname{rt}(x)=\operatorname{rt}(y) \leftrightarrow \operatorname{lt}(\varphi(x))=\operatorname{lt}(\varphi(y))
$$

whenever $x, y \in \operatorname{dom}(\varphi)$. Suppose that $\operatorname{rt}(x)=\operatorname{rt}(y)$. By symmetry, we only need to show that $\operatorname{lt}(\varphi(x)) \subseteq \operatorname{lt}(\varphi(y))$. Thus, assume that $\left(\varphi^{\prime}, \varphi^{\prime \prime}, g^{\prime}, g^{\prime \prime}\right) \in$ lt $(\varphi(x))$, where $x \in R$ and $\varphi(x) \in R^{\prime}$ for some $R, R^{\prime} \in \mathcal{R}$. Then $\varphi(x) \in$ $\operatorname{rng}\left(\varphi^{\prime}\right)$, whence by $(* *)$, there exists a $g_{1} \in G_{R}$ such that $\varphi(x)=\varphi^{\prime}\left(g_{1} x\right)$ and thus $\left(\varphi^{\prime}, \varphi^{\prime \prime}, g^{\prime}, g^{\prime \prime}\right) \in \operatorname{rt}\left(g_{1} x\right)$, while also $\left(\varphi, \varphi^{\prime}, g_{1}, 1_{R^{\prime}}\right) \in \operatorname{rt}(x)$. Taking the latter diagram to be the upper one and $\left(\varphi^{\prime}, \varphi^{\prime \prime}, g^{\prime}, g^{\prime \prime}\right) \in \operatorname{rt}\left(g_{1} x\right)$ for the lower one, we conclude from 3.5(a) that $\left(\varphi^{\prime}, \varphi^{\prime \prime}, g^{\prime}, g^{\prime \prime}\right) \in \operatorname{rt}\left(g_{1} y\right)$. But $\varphi^{\prime}\left(g_{1} y\right)=\varphi(y)$, because $\left(\varphi, \varphi^{\prime}, g_{1}, 1_{R^{\prime}}\right) \in \operatorname{rt}(y)$, which follows from $\operatorname{rt}(x)=\operatorname{rt}(y)$. Thus, finally, $\left(\varphi^{\prime}, \varphi^{\prime \prime}, g^{\prime}, g^{\prime \prime}\right) \in \operatorname{lt}(\varphi(y))$.

Now assume that $\operatorname{lt}(\varphi(x))=\operatorname{lt}(\varphi(y))$. Again, we only need to show that

$$
\left(\varphi^{\prime}, \varphi^{\prime \prime}, g^{\prime}, g^{\prime \prime}\right) \in \mathrm{rt}(x) \rightarrow\left(\varphi^{\prime}, \varphi^{\prime \prime}, g^{\prime}, g^{\prime \prime}\right) \in \mathrm{rt}(y) .
$$

Assuming $\left(\varphi^{\prime}, \varphi^{\prime \prime}, g^{\prime}, g^{\prime \prime}\right) \in \operatorname{rt}(x)$, we get the required conclusion from Lemma 3.5(b). The upper diagram is of the form $\left(\varphi, \varphi^{\prime}, 1_{R}, g_{2}\right) \in \operatorname{lt}(\varphi(x))$, and it exists by $(* *)$, since $\varphi(x)$ and $\varphi^{\prime}(x)$ must be on the same $\mathbb{G}$-orbit. Therefore $\varphi^{\prime}(x)=g_{2} \varphi(x)$, and this implies $\left(\varphi^{\prime}, \varphi^{\prime \prime}, g^{\prime}, g^{\prime \prime}\right) \in \operatorname{lt}\left(g_{2} \varphi(x)\right)$. From 3.5(b) we conclude that $\left(\varphi^{\prime}, \varphi^{\prime \prime}, g^{\prime}, g^{\prime \prime}\right) \in \operatorname{lt}\left(g_{2} \varphi(y)\right)$. Since also $\left(\varphi, \varphi^{\prime}, 1_{R}, g_{2}\right) \in$ $\operatorname{lt}(\varphi(y))$, we have $g_{2} \varphi(y)=\varphi^{\prime}(y)$, and therefore $\left(\varphi^{\prime}, \varphi^{\prime \prime}, g^{\prime}, g^{\prime \prime}\right) \in \operatorname{rt}(y)$. $\left|G_{V}\right|$.

3.9. Lemma. If $U \in \Pi, V \in \Omega, \varphi \in \Gamma_{r}^{\mathcal{R}}$ and $\varphi(U)=V$ then $\left|G_{U}\right|=$

Proof. For any $x, x^{\prime} \in U$ we see, by $(* *)$, that $x$ and $x^{\prime}$ are on the same $\mathbb{G}$-orbit iff $\varphi(x)$ and $\varphi\left(x^{\prime}\right)$ are on the same $\mathbb{G}$-orbit. So $\varphi$ maps the intersection of a $\mathbb{G}$-orbit with $U$, i.e., a $G_{U}$-orbit, onto the intersection of a $\mathbb{G}$-orbit with $V$, i.e., onto a $G_{V}$-orbit. Since the $\mathbb{G}$-actions are without fixed points, these orbits have, respectively, $\left|G_{U}\right|$ and $\left|G_{V}\right|$ elements. So $\left|G_{U}\right|=\left|G_{V}\right|$, because $\varphi$ is bijective.

This completes the proof of Theorem 3.2.

\section{A linear system without positive integral solutions}

4.1. We shall presently describe three systems $\Sigma_{A}, \Sigma_{B}, \Sigma$ of linear equations which are associated with the partitions $\Pi$ and $\Omega$ of Theorem 3.2. 
Since every $U \in \Pi$ is contained in some $R \in \mathcal{R}$, and similarly for $V \in \Omega$, we show, as in the first part of the proof of Lemma 2.12, that $U$ and $V$ are finite unions of definable rectangles. Thus it follows that the sets $U \in \Pi$ and $V \in \Omega$ are partitioned by finitely many rectangles. Let us fix, for every $U \in \Pi$ and $V \in \Omega$, some finite partitions of $U$ and $V$ into definable rectangles. We shall call the rectangles belonging to these chosen partitions $U$-selected and $V$-selected, respectively. Further, let us choose for each $U \in \Pi$ a map $\varphi \in \Gamma_{r}^{\mathcal{R}}$ such that $U \subseteq \operatorname{dom}(\varphi)$, calling such map $U$-selected. By Theorem 3.2, $\varphi(U)=V$ for some $V \in \Omega$, and since $\varphi$ splits, the image by $\varphi$ of any $U$-selected rectangle $Q \subseteq U$ is a rectangle $\varphi(Q) \subseteq V$.

For any $R \in \mathcal{R}$, consider the smallest Boolean algebra $\mathcal{B}_{A}^{R}$ of subsets of $R_{A}$ which is invariant under all bijections $g: R_{A} \rightarrow R_{A}\left(g \in G_{R}\right)$ and has among its elements the projections on $R_{A}$ of all:

(a) $U$-selected rectangles, where $U \in \Pi$ and $U \subseteq R$,

(b) $V$-selected rectangles, where $V \in \Omega$ and $V \subseteq R$,

(c) the rectangles $\varphi(Q)$, where $Q$ is a $U$-selected rectangle, $U \in \Pi$, $\varphi \in \Gamma_{r}^{\mathcal{R}}$ is the $U$-selected map and $\varphi(Q) \subseteq R$.

Let us denote by $\Xi_{A}^{R}$ the set of atoms of the (finite) Boolean algebra $\mathcal{B}_{A}^{R}$. Clearly, $\Xi_{A}^{R}$ is a $G_{R^{-}}$invariant partition of $R_{A}$ into $\mathfrak{A}$-definable sets. Let $\Xi_{A}$ be the union of all $\Xi_{A}^{R}(R \in \mathcal{R})$. We shall call the elements of $\Xi_{A}$ the horizontal atoms.

The set $\Xi_{B}$ of vertical atoms is defined analogously. It is clear that the projections (on $R_{A}$ or $R_{B}$ ) of selected rectangles and of the images of selected rectangles under selected maps are disjoint unions of atoms.

Given $R \in \mathcal{R}$, let us call any product $X \times Y$, where $X \in \Xi_{A}^{R}$ and $Y \in \Xi_{B}^{R}$, an $R$-atom, and let us denote by $\Xi^{R}$ the set of all $R$-atoms. Let further $\Xi=\bigcup\left\{\Xi^{R}: R \in \mathcal{R}\right\}$, i.e., $\Xi$ is the set of all $\mathbb{R}$-atoms. Clearly, $\Xi$ is a $\mathbb{G}$-invariant partition of $\mathbb{R}$ into definable rectangles which is a refinement of both $\Pi$ and $\Omega$. Moreover, all $U$-selected rectangles, their images under $U$-selected maps and all $V$-selected rectangles, where $U \in \Pi$ and $V \in \Omega$, are partitioned by $\mathbb{R}$-atoms.

4.2. We now associate with the partition $\Xi$ of $\mathbb{R}$ into atoms three systems $\Sigma, \Sigma_{A}, \Sigma_{B}$ of linear equations. To introduce $\Sigma$, we label the atoms $Z \in \Xi$ by variables, so that $v_{Z}$ is the variable labelling $Z$. The equations of $\Sigma$, in the variables $v_{Z}$, are such that:

(i) For each $U \in \Pi$ and every $U$-selected rectangle $Q \subseteq U$ there is in $\Sigma$ an equation whose left hand side is the sum of the variables $v_{Z}$ labelling the $\mathbb{R}$-atoms $Z \subseteq Q$, and on the right is the sum of all variables labelling the $\mathbb{R}$-atoms contained in $\varphi(Q)$, where $\varphi \in \Gamma_{r}^{\mathcal{R}}$ is the $U$-selected map. 
(ii) For each $Z \in \Xi^{R}$ and every $g \in G_{R}$ there is in $\Sigma$ the equation $v_{Z}=v_{g Z}$.

We define $\Sigma_{A}$ similarly: The horizontal atoms are labelled by variables (i.e., $\Sigma_{A}$ has $\left|\Xi_{A}\right|$ variables) and the equations are:

(j) For every $U \in \Pi$ and each $U$-selected rectangle $Q=Q_{A} \times Q_{B}$ contained in $U$ there is in $\Sigma_{A}$ an equation whose left hand side is the sum of the variables $v_{X}$ labelling the horizontal atoms $X \subseteq Q_{A}$, and the right side is the sum of all variables labelling the horizontal atoms contained in $\varphi_{A}\left(Q_{A}\right)$, where $\varphi \in \Gamma_{r}^{\mathcal{R}}$ is the $U$-selected map.

(jj) For each horizontal atom $X \in \Xi_{A}^{R}$ and every $g \in G_{R}$ there is in $\Sigma_{A}$ the equation $v_{X}=v_{g X}$.

The definition of $\Sigma_{B}$ is analogous.

Given a system of linear equations, let us call a solution of the system positive integral if this solution is obtained by assigning to every variable a suitable positive integer. Let us observe that if each of the systems $\Sigma_{A}, \Sigma_{B}$ has a positive integral solution then the same is true for $\Sigma$. Indeed, if we have such solutions for $\Sigma_{A}$ and $\Sigma_{B}$, we can assign to every $v_{Z}$, where $Z=X \times Y \in \Xi$, the product of the (positive integral) values assigned by these solutions to $v_{X}$ and $v_{Y}$; it is not hard to see that this gives a positive integral solution for $\Sigma$.

Our next purpose is to show that $\Sigma$ has no positive integral solution. Consequently, one of the systems $\Sigma_{A}, \Sigma_{B}$ cannot have a positive integral solution, and this fact will be used to show that $\mathbf{T}$ interprets in a disjoint product $\mathfrak{S} \dot{\times} \mathfrak{A}$ or $\mathfrak{S} \dot{\times} \mathfrak{B}$, for some finite structure $\mathfrak{S}$.

4.3. TheOREM. The system $\Sigma$ has no positive integral solution.

Pr o of. Suppose there is a positive integral solution for $\Sigma$. Since the system $\Sigma$ is homogeneous, we may assume that all the positive integral values which constitute the solution are divisible by all orders of the stabilizers $G_{Z}$ of the atoms $Z \in \Xi$. Let us now assign to every atom $Z \in \Xi$ a finite set $\bar{Z}$ such that the number of elements $|\bar{Z}|$ of that set is equal to the positive value assigned in the solution of $\Sigma$ to the variable $v_{Z}$. Further, let this assignment be such that disjoint sets are assigned to different atoms. We now replace every atom $Z \in \Xi$ by the corresponding finite set $\bar{Z}$. In detail, we put $\bar{\Xi}=\{\bar{Z}: Z \in \Xi\}$, and more generally, if $S \subseteq \mathbb{R}$ is any set which is a union of atoms, or $\mathcal{F}$ is a family of subsets of $\mathbb{R}$, where each $F \in \mathcal{F}$ is a union of atoms, then we abbreviate:

$$
\bar{S}=\bigcup\{\bar{Z}: Z \subseteq S \wedge Z \in \Xi\}, \quad \overline{\mathcal{F}}=\{\bar{F}: F \in \mathcal{F}\} .
$$

So, replacing each atom $Z$ by $\bar{Z}$, we get:

$$
\overline{\mathbb{R}}=\text { disjoint union of all } \bar{Z} \in \bar{\Xi},
$$


$\bar{R}=$ disjoint union of all $\bar{Z} \in \bar{\Xi}$ such that $Z \subseteq R(R \in \mathcal{R})$,

$\overline{\mathcal{R}}=$ partition of $\overline{\mathbb{R}}$ by the subsets $\bar{R}$, where $R \in \mathcal{R}$,

$\bar{U}=$ disjoint union of all $\bar{Z} \in \bar{\Xi}$ such that $Z \subseteq U(U \in \Pi)$,

$\bar{\Pi}=$ partition of $\overline{\mathbb{R}}$ by the subsets $\bar{U}$, where $U \in \Pi$,

$\bar{V}=$ disjoint union of all $\bar{Z} \in \bar{\Xi}$ such that $Z \subseteq V(V \in \Omega)$,

$\bar{\Omega}=$ partition of $\overline{\mathbb{R}}$ by the subsets $\bar{V}$, where $V \in \Omega$.

A consequence of these definitions is that for each $\bar{U} \in \bar{\Pi}$ and $\bar{V}=\overline{\varphi(U)}$, where $\varphi \in \Gamma_{r}^{\mathcal{R}}$ is the $U$-selected map, one gets $|\bar{U}|=|\bar{V}|$. Indeed, this follows from the equations of $\Sigma$, strictly speaking, from the equations of type 4.2(i) determined by $U$ and $\varphi$.

The $\mathbb{G}$-action on $\Xi$, resulting from the $\mathbb{G}$-invariance of $\Xi$, determines via the obvious bijection $\Xi \rightarrow \bar{\Xi}$ a $\mathbb{G}$-action on $\bar{\Xi}$ (thus given by $g \bar{Z}=\overline{g Z}$ ). Our first purpose is to extend this $\mathbb{G}$-action on $\bar{\Xi}$ to a $\mathbb{G}$-action on $\overline{\mathbb{R}}$ so that for every $R \in \mathcal{R}, G_{R}$ acts without fixed points on $\bar{R}$. The given $\mathbb{G}$-action on $\bar{\Xi}$ consists of $G_{R^{-a c t i o n s}}$ on the various families of sets $\bar{\Xi}^{R}$ and every $\mathbb{G}$-orbit in $\bar{\Xi}$ is a $G_{R^{-}}$orbit for some $R \in \mathcal{R}$. Let us select one representative for every $G_{R^{-o r b i t}}$ in $\bar{\Xi}^{R}$ and denote by $\bar{\Xi}_{0}^{R}$ the set of these representatives.

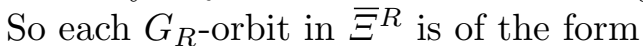

$(\odot) \quad\left\{\bar{Z}_{0}, g_{1} \bar{Z}_{0}, \ldots, g_{m} \bar{Z}_{0}\right\} \quad$ for some $\bar{Z}_{0} \in \bar{\Xi}_{0}^{R}$ and $g_{1}, \ldots, g_{m} \in G_{R}$.

Since $G_{Z_{0}}$ is the stabilizer of $\bar{Z}_{0}$, it is clear that $\left\{G_{Z_{0}}, g_{1} G_{Z_{0}}, \ldots, g_{m} G_{Z_{0}}\right\}$ is the set of right $G_{Z_{0}}$-cosets in $G_{R}$. Obviously we can imagine $\bar{Z}_{0}$ to be a disjoint union of copies of $G_{Z_{0}}$ (which is possible because $\left|\bar{Z}_{0}\right|$ is divisible by the orders of all stabilizers of atoms). Next, observing that the sets $\bar{Z}_{0}, g_{1} \bar{Z}_{0}, \ldots, g_{m} \bar{Z}_{0}$ have the same number of elements, in view of the equations in $\Sigma$ of type $4.2\left(\right.$ ii), we can think of any $g_{j} \bar{Z}_{0}$ as obtained from $\bar{Z}_{0}$ by replacing each copy of $G_{Z_{0}}$ contained in $\bar{Z}_{0}$ by the coset $g_{j} G_{Z_{0}}$. In this way we can regard

$$
\bar{Z}_{0} \cup g_{1} \bar{Z}_{0} \cup \ldots \cup g_{m} \bar{Z}_{0}
$$

as a disjoint union of copies of $G_{R}$. Letting $G_{R}$ act on each of its copies by left multiplication, we get a $G_{R}$-action on the above union which induces the originally given $\mathbb{G}$-action on the $\mathbb{G}$-orbit $(\odot)$ in $\bar{\Xi}$.

If, for every $G_{R}$-orbit $(\odot)$, we apply the above definition to the corresponding set $(\odot)$, we get a $G_{R}$-action on $\bar{R}$. Repeating the same for all $\bar{R} \in \overline{\mathcal{R}}$, we get a $\mathbb{G}$-action on $\overline{\mathbb{R}}$. Calling the various $G_{R^{-}}$orbits in $\overline{\mathbb{R}}$ $\mathbb{G}$-orbits and denoting by $\overline{\mathbb{R}} / \mathbb{G}$ the set of these $\mathbb{G}$-orbits, we shall now show that there is a non-surjective injection $\Phi: \overline{\mathbb{R}} / \mathbb{G} \rightarrow \overline{\mathbb{R}} / \mathbb{G}$. Since $\overline{\mathbb{R}}$ is finite, this will provide the required contradiction.

Under the $\mathbb{G}$-action on $\overline{\mathbb{R}}$ just defined, for every $\bar{U} \in \bar{\Pi}$, each image $g \bar{U}$ with $g \in \mathbb{G}$ is determined by the images $g \bar{Z}=\overline{g Z}$ of all $\bar{Z} \subseteq \bar{U}, \bar{Z} \in \bar{\Xi}$. 
Since a similar property holds for all $\bar{V} \in \bar{\Omega}$, it follows that

$(\oplus) \quad g \bar{U}=\overline{g U}$ and $g \bar{V}=\overline{g V}$ for all $U \in \Pi, V \in \Omega, g \in \mathbb{G}$,

i.e., $\bar{\Pi}$ and $\bar{\Omega}$ are $\mathbb{G}$-invariant partitions of $\overline{\mathbb{R}}$. Thus we now have three $\mathbb{G}$-actions: on $\overline{\mathbb{R}}$, on $\bar{\Pi}$ and on $\bar{\Omega}$. The three corresponding sets of $\mathbb{G}$-orbits will be denoted by $\overline{\mathbb{R}} / \mathbb{G}, \bar{\Pi} / \mathbb{G}$ and $\bar{\Omega} / \mathbb{G}$. We conclude from $(\oplus)$ that the stabilizer of $\bar{U} \in \bar{\Pi}$ (with respect to the $\mathbb{G}$-action on $\bar{\Pi}$ ) is $G_{U}$, and similarly, that $G_{V}$ is the stabilizer of $\bar{V} \in \bar{\Omega}$. Let $V=\varphi(U)$, where $\varphi \in \Gamma_{r}^{\mathcal{R}}$ is the $U$-selected map. Then, as we have shown above, $\left|G_{U}\right|=\left|G_{V}\right|$ and $|\bar{U}|=|\bar{V}|$. Thus the number of $G_{U}$-orbits in $\bar{U}$ is the same as the number of $G_{V}$-orbits in $\bar{V}$. So, if $\bar{U} / G_{U}$ and $\bar{V} / G_{V}$ denote the sets of these orbits then we have a bijection $\Phi_{U}: \bar{U} / G_{U} \rightarrow \bar{V} / G_{V}$.

To define the required $\Phi: \overline{\mathbb{R}} / \mathbb{G} \rightarrow \overline{\mathbb{R}} / \mathbb{G}$, let us select one representative $\bar{U}_{0} \in \bar{\Pi}$ from every $\mathbb{G}$-orbit $\mathbb{G} \bar{U} \in \bar{\Pi} / \mathbb{G}$, and let $\bar{\Pi}_{0} \subseteq \bar{\Pi}$ denote the set of these representatives. Then every orbit $\mathbb{G} x \in \overline{\mathbb{R}} / \mathbb{G}(x \in \overline{\mathbb{R}})$ intersects exactly one $\bar{U}_{0} \in \bar{\Pi}_{0}$, and that intersection is a $G_{U_{0}}$-orbit in $\bar{U}_{0}$. Similarly, the non-empty intersections of orbits $\mathbb{G} x \in \overline{\mathbb{R}} / \mathbb{G}$ with any $\bar{V} \in \bar{\Omega}$ are the $G_{V}$-orbits in $\bar{V}$. For any $\mathbb{G} x \in \overline{\mathbb{R}} / \mathbb{G}$ we now define $\Phi(\mathbb{G} x) \in \mathbb{R} / \mathbb{G}$ as follows. First, we assign to $\mathbb{G} x$ the unique non-empty intersection $\mathbb{G} x \cap \bar{U}_{0}$, where $\bar{U}_{0} \in \bar{\Pi}_{0}$. (This is a $G_{U_{0}}$-orbit in $\bar{U}_{0}$.) Next, we consider the $U_{0}$-selected map $\varphi_{0} \in \Gamma_{r}^{\mathcal{R}}$ and put $V_{0}=\varphi_{0}\left(U_{0}\right)$. Then, taking the image of the $G_{U_{0}}$-orbit $\mathbb{G} x \cap \bar{U}_{0}$ by the map $\Phi_{U_{0}}$ introduced above, we get the $G_{V_{0}}$-orbit $\Phi_{U_{0}}\left(\mathbb{G} x \cap \bar{U}_{0}\right)$ in $\bar{V}_{0}$. Finally, we define $\Phi(\mathbb{G} x)$ to be the orbit $\mathbb{G} x^{\prime} \in \overline{\mathbb{R}} / \mathbb{G}$ which intersects $\bar{V}_{0}$ along $\Phi_{U_{0}}\left(\mathbb{G} x \cap \bar{U}_{0}\right)$.

We claim that $\Phi$ is injective. This is clearly so on the subset of those orbits $\mathbb{G} x \in \overline{\mathbb{R}} / \mathbb{G}$ which meet one fixed $\bar{U}_{0} \in \bar{\Pi}_{0}$. On the other hand, if $\bar{U}_{0}, \bar{U}_{0}^{\prime} \in \bar{\Pi}_{0}$ are distinct then no orbit $\mathbb{G} x \in \overline{\mathbb{R}} / \mathbb{G}$ meets both $\bar{U}_{0}$ and $\bar{U}_{0}^{\prime}$, and hence no $\mathbb{G}$-orbit in $\mathbb{R}$ meets both $U_{0}, U_{0}^{\prime} \in \Pi$ (see $(\oplus)$ ). Thus, if $\varphi, \varphi^{\prime} \in \Gamma_{r}^{\mathcal{R}}$ are the $U_{0}$-selected and $U_{0}^{\prime}$-selected maps, then by $3.3(* *)$, no $\mathbb{G}$-orbit in $\mathbb{R}$ meets both $V_{0}=\varphi\left(U_{0}\right)$ and $V_{0}^{\prime}=\varphi^{\prime}\left(U_{0}^{\prime}\right)$. But then no orbit $\mathbb{G} x \in \overline{\mathbb{R}} / \mathbb{G}$ meets both $\bar{V}_{0}$ and $\bar{V}_{0}^{\prime}$, by $(\oplus)$. This proves the injectivity of $\Phi$.

By the last part of Theorem 3.2, every $\mathbb{G}$-orbit in $\mathbb{R}$ which meets $V_{*} \in \Omega$ is disjoint from all $\varphi(U)\left(U \in \Pi, \varphi \in \Gamma_{r}^{\mathcal{R}}\right)$. Hence, by $(\oplus)$, every orbit $\mathbb{G} x \in \overline{\mathbb{R}} / \mathbb{G}$ which meets $\bar{V}_{*}$ is disjoint from all $\overline{\varphi_{0}\left(U_{0}\right)}$, where $\bar{U}_{0} \in \bar{\Pi}_{0}$ and $\varphi_{0} \in \Gamma_{r}^{\mathcal{R}}$ are as above, and thus no such orbit $\mathbb{G} x$ is in the image of $\Phi$.

In the next section (referring to the theorem proved in the Appendix) we give the last part of the proof of Theorem 1.3.

\section{Conclusion of the proof}

5.1. As we observed in 4.2 , Theorem 4.3 implies that one of the systems $\Sigma_{A}, \Sigma_{B}$, say $\Sigma_{A}$, has no positive integral solution. By the result given 
in the Appendix, there exists a system of equations $\left(e_{1}, \ldots, e_{m}\right)$ which is reflection equivalent to a subsystem of $\Sigma_{A}$ and right unbalanced. We put $\bar{m}=\{1, \ldots, m\}$ and for any $i \in \bar{m}$ denote by $\mathbf{l}(i)$ the disjoint union of horizontal atoms $X$ such that $v_{X}$ occurs on the left side of $e_{i}$, and by $\mathbf{r}(i)$ the disjoint union of horizontal atoms $X$ such that $v_{X}$ occurs on the right side of $e_{i}$.

By the definition of $\Sigma_{A}$ in 4.2 , for any $i \in \bar{m}$ there is a $\varphi \in \Gamma_{r}^{\mathcal{R}}$ such that either $\mathbf{l}(i) \subseteq \operatorname{dom}\left(\varphi_{A}\right)$ and $\mathbf{r}(i) \subseteq \operatorname{rng}\left(\varphi_{A}\right)$, or $\mathbf{l}(i) \subseteq \operatorname{rng}\left(\varphi_{A}\right)$ and $\mathbf{r}(i) \subseteq \operatorname{dom}\left(\varphi_{A}\right)$. So, in any case there exist $R, R^{\prime} \in \mathcal{R}$ such that $\mathbf{l}(i) \subseteq R_{A}$ and $\mathbf{r}(i) \subseteq R_{A}^{\prime}$, whence

$$
\mathbf{l}(i) \subseteq D_{A}=\bar{D}_{A} / \bar{\varepsilon}_{A}^{D} \quad \text { for some } \bar{D} \in \overline{\mathcal{D}}_{\varepsilon},
$$

and similarly $\mathbf{r}(i) \subseteq \bar{D}_{A}^{\prime} / \bar{\varepsilon}_{A}^{D^{\prime}}$ for some $\bar{D}^{\prime} \in \overline{\mathcal{D}}_{\varepsilon}$ (see 2.2 and 2.3). Hence, if $\lambda_{i}$ denotes the equivalence relation on $A$ which coincides with $\bar{\varepsilon}_{A}^{D}$ on $\bar{D}_{A} \subseteq A$ and with equality outside $\bar{D}_{A}$ then $\mathbf{l}(i) \subseteq A / \lambda_{i}$. Similarly, we have $\mathbf{r}(i) \subseteq$ $A / \varrho_{i}$ for the equivalence relation $\varrho_{i}$ on $A$ which coincides with $\bar{\varepsilon}_{A}^{D^{\prime}}$ on $\bar{D}_{A}^{\prime} \subseteq$ $A$ and with equality outside $\bar{D}_{A}^{\prime}$. Clearly, both $\lambda_{i}$ and $\varrho_{i}$ are $\mathfrak{A}$-definable. We shall denote by $\gamma_{i}$ the bijection $\mathbf{l}(i) \rightarrow \mathbf{r}(i)$ which arises by restricting $\varphi_{A}$ or $\varphi_{A}^{-1}$, as the case may be, to $\mathbf{l}(i)$. Then $\gamma_{i}$ is $\mathfrak{A}$-definable in the sense of 2.5 (i.e., its graph $\bar{\gamma}_{i} \subseteq A / \lambda_{i} \times A / \varrho_{i}$ determines an $\mathfrak{A}$-definable subset $\lambda_{i} \cdot \bar{\gamma}_{i} \cdot \varrho_{i}$ of $\left.A^{2}\right)$.

A finite structure $\mathfrak{S}$ and an equivalence relation $\simeq$ on $\mathfrak{S} \dot{\times} \mathfrak{A}$ are now defined as follows. The universe $S$ of $\mathfrak{S}$ is the union of two disjoint sets $S_{\mathfrak{l}}$ and $S_{\mathfrak{r}}$ such that $\left|S_{\mathfrak{l}}\right|=\left|S_{\mathfrak{r}}\right|=m$, and the only defining relation of $\mathfrak{S}$ is $=$. We now assign to every $i \in \bar{m}$ an element $\mathfrak{l}_{i} \in S_{\mathfrak{l}}$ and an element $\mathfrak{r}_{i} \in S_{\mathfrak{r}}$ so that the resulting maps from $\bar{m}$ to $S_{\mathfrak{l}}$ and $S_{\mathfrak{r}}$ are bijective. The equivalence relation $\simeq$ on $S \times A$ is defined by requiring that for every $s, s^{\prime} \in S$ and $a, a^{\prime} \in A$,

$$
\begin{aligned}
(s, a) \simeq\left(s^{\prime}, a^{\prime}\right) \leftrightarrow & \left(s=s^{\prime}=\mathfrak{l}_{i} \text { and } \lambda_{i}\left(a, a^{\prime}\right) \text { for some } i \in \bar{m}\right) \\
& \vee\left(s=s^{\prime}=\mathfrak{r}_{i} \text { and } \varrho_{i}\left(a, a^{\prime}\right) \text { for some } i \in \bar{m}\right) .
\end{aligned}
$$

Clearly $\simeq$ is $(\mathfrak{S} \dot{\times} \mathfrak{A})$-definable.

5.2. We shall now describe two subsets $\mathbb{K}_{\mathfrak{l}}, \mathbb{K}_{\mathfrak{r}}$ of $(S \times A) / \simeq$, a bijection $\gamma: \mathbb{K}_{\mathfrak{l}} \rightarrow \mathbb{K}_{\mathfrak{r}}$, and a non-surjective injection $\psi: \mathbb{K}_{\mathfrak{l}} \rightarrow \mathbb{K}_{\mathfrak{r}}$, all of them definable in $(\mathfrak{S} \dot{\times} \mathfrak{A}) / \simeq$. This will end the proof of Theorem 1.3, since then the map of $(S \times A) / \simeq$ into itself which is equal to $\psi \gamma^{-1}$ on $\mathbb{K}_{\mathfrak{r}}$ and is the equality outside $\mathbb{K}_{\mathfrak{r}}$, is a non-surjective injection.

For every $i \in \bar{m}$ let us make the following abbreviations: if $Z \subseteq A / \lambda_{i}$, we shall denote the subset $\left\{\mathfrak{l}_{i}\right\} \times Z$ of $(S \times A) / \simeq$ by $\left(\mathfrak{l}_{i}, Z\right)$. Similarly, for $Z^{\prime} \subseteq A / \varrho_{i}$ we put $\left(\mathfrak{r}_{i}, Z^{\prime}\right)=\left\{\mathfrak{r}_{i}\right\} \times Z^{\prime}$.

The sets $\mathbb{K}_{\mathfrak{l}}, \mathbb{K}_{\mathfrak{r}} \subseteq(S \times A) / \simeq$ are the disjoint unions

$$
\mathbb{K}_{\mathfrak{l}}=\bigcup\left\{\left(\mathfrak{l}_{i}, \mathbf{l}(i)\right): i \in \bar{m}\right\}, \quad \mathbb{K}_{\mathfrak{r}}=\bigcup\left\{\left(\mathfrak{r}_{i}, \mathbf{r}(i)\right): i \in \bar{m}\right\} .
$$


Evidently $\mathbb{K}_{\mathfrak{l}}$ and $\mathbb{K}_{\mathfrak{r}}$ are definable in $(\mathfrak{S} \dot{\times} \mathfrak{A}) / \simeq$. Each bijection $\gamma_{i}$ yields a bijection $\left(\mathfrak{l}_{i}, \mathbf{l}(i)\right) \rightarrow\left(\mathfrak{r}_{i}, \mathbf{r}(i)\right)$ given by

$$
\left(\mathfrak{l}_{i}, a / \lambda_{i}\right) \mapsto\left(\mathfrak{r}_{i}, \gamma_{i}\left(a / \lambda_{i}\right)\right) .
$$

The union of these bijections over all $i \in \bar{m}$ is a bijection $\gamma: \mathbb{K}_{\mathfrak{l}} \rightarrow \mathbb{K}_{\mathfrak{r}}$ which is clearly definable in $(\mathfrak{S} \dot{\times} \mathfrak{A}) / \simeq$.

For $i \in \bar{m}$ and any horizontal atom $X$ such that $v_{X}$ occurs on the left side of $e_{i}$, one has $X \subseteq A / \lambda_{i}$ and thus $\left(\mathfrak{l}_{i}, X\right) \subseteq \mathbb{K}_{\mathfrak{r}}$. Let us call every such set $\left(\mathfrak{l}_{i}, X\right)$ a left atom, or more precisely, an $X$-based left atom. It is clear that the left atoms form a partition of $\mathbb{K}_{\mathfrak{r}}$. Similarly, $\mathbb{K}_{\mathfrak{r}}$ is partitioned by the sets $\left(\mathfrak{r}_{i}, X\right)$, where $i \in \bar{m}$ and $X$ is such that $v_{X}$ occurs on the right side of $e_{i}$. We shall call every such $\left(\mathfrak{r}_{i}, X\right)$ an $X$-based right atom.

Using left and right atoms, we now define a non-surjective injection $\psi: \mathbb{K}_{\mathfrak{r}} \rightarrow \mathbb{K}_{\mathfrak{r}}$. Since $\left(e_{1}, \ldots, e_{m}\right)$ is right unbalanced, it follows that for every $X \in \Xi_{A}$ the number of $X$-based left atoms does not exceed the number of $X$-based right atoms. Also, there is at least one $X_{0} \in \Xi_{A}$ such that the number of $X_{0}$-based left atoms is strictly less than the number of $X_{0}$-based right atoms. So there is a non-surjective injection $\Psi$ from the set of left atoms to the set of right atoms which assigns, for any $X \in \Xi_{A}$, to every $X$-based left atom an $X$-based right atom. To any $X$-based atoms $\left(\mathfrak{l}_{i}, X\right),\left(\mathfrak{r}_{j}, X\right)$ such that $\Psi\left(\left(\mathfrak{l}_{i}, X\right)\right)=\left(\mathfrak{r}_{j}, X\right)$ let us assign the bijection $\left(\mathfrak{l}_{i}, X\right) \rightarrow\left(\mathfrak{r}_{j}, X\right)$ given by the identity map on $X$. The union of these bijections, taken for all $i \in \bar{m}$, is an injection $\psi: \mathbb{K}_{\mathfrak{l}} \rightarrow \mathbb{K}_{\mathfrak{r}}$. This map is not surjective, since $\Psi$ is not surjective. It is clear that $\psi$ is definable in $(\mathfrak{S} \dot{\times} \mathfrak{A}) / \simeq$.

Appendix: linear systems with no positive solution. A system $\Sigma=\left(e_{1}, \ldots, e_{m}\right)$ of linear equations

$$
e_{i}: \quad \sum_{j=1}^{n} a_{i j} v_{j}=\sum_{j=1}^{n} b_{i j} v_{j}, \quad i=1, \ldots, m,
$$

in the variables $v_{1}, \ldots, v_{n}$ will be called primitive if $a_{i j}, b_{i j} \in\{0,1\}$ for all $i, j$. Thus each of the sides of any equation $e_{i}$ is a sum of some variables selected from among $v_{1}, \ldots, v_{n}$. By a positive solution of $\Sigma$ we shall mean a solution in which all the variables take positive values. We shall be mainly concerned with solutions composed of positive integers, calling them positive integral solutions. Our purpose is to characterize those primitive systems which have no positive integral solution. To state the result, let us denote by $\bar{e}_{i}$ the reflection of an equation $e_{i}$, i.e., for $e_{i}$ as above,

$$
\bar{e}_{i}: \quad \sum_{j=1}^{n} b_{i j} v_{j}=\sum_{j=1}^{n} a_{i j} v_{j} .
$$

Further, let us call $\Sigma$ right unbalanced if every variable $v_{j}$ occurs at least as 
many times on the right sides of the equations of $\Sigma$ as on the left sides, and there is a variable, say $v_{j_{0}}$, which occurs more times on the right sides than on the left sides:

$$
\sum_{i=1}^{m} a_{i j_{0}}<\sum_{i=1}^{m} b_{i j_{0}} \quad \text { and } \quad \sum_{i=1}^{m} a_{i j} \leq \sum_{i=1}^{m} b_{i j} \quad \text { for } j=1, \ldots, n .
$$

(A system which is not right unbalanced might become such after some equations of the system are repeated; thus, to allow for repetitions, we do not consider sets, but rather systems of equations.) By interchanging in the above definition the words "right" and "left" we get the definition of a left unbalanced system. If a system is right or left unbalanced, we shall simply call it unbalanced.

Let us call two primitive systems reflection equivalent if for each equation $e$ of one system, either $e$ or its reflection $\bar{e}$ is in the other system. It is evident that a system is reflection equivalent to a left unbalanced system iff it is reflection equivalent to a right unbalanced one.

THEOREM. A primitive system $\Sigma$ has no positive integral solution iff $\Sigma$ contains a subsystem which is reflection equivalent to an unbalanced system $\Sigma_{*}$.

The sufficiency of the condition requires no proof. The proof of necessity, which follows now, has been suggested by Alan Stern. (Our original proof was not self-contained: it used a separation theorem for convex sets in Euclidean space.) Since no part of this proof is directly linked to any other part of the paper, we shall not be concerned henceforth about keeping the notation in agreement with the previous sections.

Let $\mathbb{Q}^{n}$ be the $n$-dimensional vector space over the field $\mathbb{Q}$ of rational numbers. The main step in the proof is a lemma concerning a pair of orthogonal complementary subspaces $V, W \subseteq \mathbb{Q}^{n}$, i.e., such that $V \perp W$ and $V+W=\mathbb{Q}^{n}$. To state it, let us call a vector $x \in \mathbb{Q}^{n}$ non-negative (resp. positive) if all components of $x$ are non-negative (resp. positive). These properties will be abbreviated as $x \geq 0$ and $x>0$.

Lemma (A. S. Stern). If $V, W$ are orthogonal complementary subspaces of $\mathbb{Q}^{n}$ and $V$ contains no positive vector then $W$ contains a non-zero, nonnegative vector.

Proof (by induction on $n$ ). If $n=1$ then $V=\mathbb{Q}$ or $W=\mathbb{Q}$ and the lemma is obvious. So let $n>1$ and let us identify the space $\mathbb{Q}^{n-1}$ with a subspace of $\mathbb{Q}^{n}$ so that the map $\pi$ given by

$$
\pi\left(x_{1}, \ldots, x_{n-1}, x_{n}\right)=\left(x_{1}, \ldots, x_{n-1}, 0\right)
$$

projects $\mathbb{Q}^{n}$ onto $\mathbb{Q}^{n-1}$. We adopt the inductive hypothesis that the lemma holds for orthogonal complementary subspaces of $\mathbb{Q}^{n-1}$. 
Let $V, W$ be orthogonal complementary subspaces of $\mathbb{Q}^{n}$, and let us assume that $V$ does not contain a positive vector. Our purpose is to show that there is a $w \in W$ such that $0 \neq w \geq 0$.

If $V \subseteq \mathbb{Q}^{n-1}$ then $w=(0, \ldots, 0,1) \in W$ satisfies $0 \neq w \geq 0$ and we are done. So let us assume that $V \nsubseteq \mathbb{Q}^{n-1}$. We put $V^{\prime}=V \cap \mathbb{Q}^{n-1}$, $W^{\prime}=W \cap \mathbb{Q}^{n-1}$ and leave it to the reader to check that $V^{\prime}$ and $\pi(W)$ are orthogonal complementary subspaces of $\mathbb{Q}^{n-1}$; likewise $W^{\prime}$ and $\pi(V)$. To apply the inductive assumption to each of the pairs $V^{\prime}, \pi(W)$ and $W^{\prime}$, $\pi(V)$, let us call a vector $x$ positive in $\mathbb{Q}^{n-1}$ if $x \in \mathbb{Q}^{n-1}$ and the first $n-1$ components of $x$ are positive.

Let us show first that there is a non-zero, non-negative vector in $\pi(W)$. By the inductive hypothesis, it will be enough to check that $V^{\prime}$ does not contain a vector positive in $\mathbb{Q}^{n-1}$. Since $V \nsubseteq \mathbb{Q}^{n-1}$ it follows that for some $u \in V$ we have $u_{n}>0$, so if $V^{\prime}$ were to contain a vector $x$ positive in $\mathbb{Q}^{n-1}$, we would get

$$
x+\varepsilon u=\left(x_{1}+\varepsilon u_{1}, \ldots, x_{n-1}+\varepsilon u_{n-1}, \varepsilon u_{n}\right) \in V
$$

for all $\varepsilon \in \mathbb{Q}$. Taking $\varepsilon>0$ small enough, we would obtain a positive $x+\varepsilon u \in V$, a contradiction. So $V^{\prime}$ does not contain a vector positive in $\mathbb{Q}^{n-1}$, whence there is a $y \in \pi(W)$ satisfying $0 \neq y \geq 0$.

Now either there is a non-zero non-negative vector in $W^{\prime}$, in which case we are done, since $W^{\prime} \subseteq W$, or there is no such vector. In the latter case, by the inductive hypothesis, there is a $z \in \pi(V)$, positive in $\mathbb{Q}^{n-1}$, say $z=\pi(v)$, where $v \in V$. Since $v>0$ is excluded, we must have $v_{n} \leq 0$. We have shown above that $0 \neq y \geq 0$ for some $y \in \pi(W)$, say $y=\pi(w)$, where $w \in W$. Since $\pi(v)$ is positive in $\mathbb{Q}^{n-1}$ and $0 \neq \pi(w) \geq 0$, the dot product $\pi(v) \cdot \pi(w)$ is positive. But $v \perp w$, whence

$$
0=v \cdot w=\pi(v) \cdot \pi(w)+v_{n} w_{n} .
$$

This implies that $v_{n} w_{n}<0$, and since we know that $v_{n} \leq 0$, it follows that $w_{n}>0$. Hence $w$ is the required non-negative vector in $W$.

Proof of the Theorem. Assume that $\Sigma$ has no positive integral solution, and consider the $m \times n$ matrices $A=\left(a_{i j}\right), B=\left(b_{i j}\right)$ and $C=A-B$. Then the linear system represented by the matrix equation $C x=0$ has no positive integral solution. Let $W$ be the subspace of $\mathbb{Q}^{n}$ spanned by the rows of $C$ and let $V$ be the orthogonal complement in $\mathbb{Q}^{n}$ of $W$. Then $x \in V$ iff $C x=0$, whence there is no positive integral vector in $V$. Consequently, there is no positive vector in $V$, and thus, by the Lemma, there is a $w \in W$ satisfying $0 \neq w \geq 0$. Let us write $w$ as a linear combination of the rows of $C$, say with coefficients $\alpha_{1}, \ldots, \alpha_{m} \in \mathbb{Q}$.

We may assume that $\alpha_{1}, \ldots, \alpha_{m} \geq 0$. Indeed, if some $\alpha_{i}$ are negative, we can consider instead of $\Sigma$ the system obtained by replacing in $\Sigma$ the corresponding equations $e_{i}$ by their reflections $\bar{e}_{i}$. 
We can also assume that $\alpha_{1}, \ldots, \alpha_{m}$ are integers. Indeed, if they are not, let $\beta>0$ be the product of their denominators. Then $\beta w \in W, 0 \neq \beta w \geq 0$ and the integers $\beta \alpha_{1}, \ldots, \beta \alpha_{m}$ are the coefficients of a linear combination of rows of $C$ which is equal to $\beta w$.

The required unbalanced system $\Sigma_{*}$ is now obtained from $\Sigma$ by replacing each equation $e_{i}$ by $\alpha_{i}$ copies of $e_{i}(i=1, \ldots, m)$. We note that then each $w_{j}$ equals the number of occurrences of $v_{j}$ on the left sides of the equations of $\Sigma_{*}$ minus the number of occurrences of $v_{j}$ on the right sides of the equations of $\Sigma_{*}$. So it follows from $0 \neq w \geq 0$ that $\Sigma_{*}$ is left unbalanced.

Added in proof (October 1995). Stern's lemma has a neat generalization: If $W$ and $V$ are finitely generated convex cones in $\mathbb{Q}^{n}$ which are polars of each other and $V$ contains no positive vector then $W$ contains a non-zero non-negative vector. The only place where the above proof changes essentially is in checking that $V^{\prime}$ does not contain a vector positive in $\mathbb{Q}^{n-1}$. Consider first the case where for every $u \in V$ we have $u_{n} \leq 0$. Then $(0, \ldots, 0,1) \in W$, so we are done. Alternatively, if there is a $u \in V$ with $u_{n}>0$, the proof continues as above. [This generalization might come useful if one would attempt to solve, by the methods of this paper, the dual problem posed in the introduction.]

\section{References}

[M] J. Mycielski, A lattice connected with relative interpretability of theories, Notices Amer. Math. Soc. 9 (1962), 407-408; Erratum, ibid. 18 (1971), 984.

[MPS] J. Mycielski, P. Pudlák and A. Stern, A lattice of chapters of mathematics, Mem. Amer. Math. Soc. 426 (1991).

[S] A. S. Stern, Sequential theories and infinite distributivity in the lattice of chapters, J. Symbolic Logic 54 (1989), 190-206.

[SS] A. S. Stern and S. S. Świerczkowski, A class of connected theories of order, ibid. 59 (1994), 534-542.

DEPARTMENT OF MATHEMATICS AND STATISTICS

SULTAN QABOOS UNIVERSITY

P.O.BOX 36, AL-KHOD

123 MUSCAT, SULTANATE OF OMAN

E-mail: SCW0307@SQU.EDU 